\title{
Neutrino and antineutrino inclusive charged-current cross section measurements with the MINOS near detector
}

P. Adamson, ${ }^{8}$ C. Andreopoulos, ${ }^{19}$ K. E. Arms,${ }^{14}$ R. Armstrong, ${ }^{12}$ D. J. Auty,${ }^{23}$ D. S. Ayres,${ }^{1}$ C. Backhouse, ${ }^{17}$ P. D. Barnes, Jr., ${ }^{29}$ G. Barr, ${ }^{17}$ W. L. Barrett, ${ }^{30}$ D. Bhattacharya, ${ }^{18}$ M. Bishai, ${ }^{4}$ A. Blake, ${ }^{6}$ G. J. Bock, ${ }^{8}$ D. J. Boehnlein, ${ }^{8}$

D. Bogert, ${ }^{8}$ C. Bower, ${ }^{12}$ S. Cavanaugh, ${ }^{9}$ J. D. Chapman, ${ }^{6}$ D. Cherdack, ${ }^{26}$ S. Childress, ${ }^{8}$ B. C. Choudhary, ${ }^{8,5}, *$ J. A. B. Coelho, ${ }^{7}$ S. J. Coleman, ${ }^{28}$ D. Cronin-Hennessy, ${ }^{14}$ A. J. Culling, ${ }^{6}$ I. Z. Danko, ${ }^{18}$ J. K. de Jong, ${ }^{17,11}$ N. E. Devenish, ${ }^{23}$ M. V. Diwan, ${ }^{4}$ M. Dorman, ${ }^{13,19}$ A. R. Erwin, ${ }^{31}$ C. O. Escobar, ${ }^{7}$ J. J. Evans, ${ }^{13,17}$ E. Falk, ${ }^{23}$ G. J. Feldman, ${ }^{9}$ M. V. Frohne, ${ }^{10,3}$ H. R. Gallagher, ${ }^{26}$ A. Godley, ${ }^{21}$ M. C. Goodman, ${ }^{1}$ P. Gouffon, ${ }^{20}$ R. Gran, ${ }^{15}$ E. W. Grashorn,,${ }^{14,15}$ K. Grzelak, ${ }^{27,17}$ A. Habig, ${ }^{15}$ D. Harris,${ }^{8}$ P. G. Harris,${ }^{23}$ J. Hartnell, ${ }^{23,19}$ R. Hatcher, ${ }^{8}$ K. Heller,${ }^{14}$ A. Himmel, ${ }^{5}$ A. Holin, ${ }^{13}$ J. Hylen, ${ }^{8}$ G. M. Irwin, ${ }^{22}$ Z. Isvan, ${ }^{18}$ D. E. Jaffe ${ }^{4}$ C. James, ${ }^{8}$ D. Jensen, ${ }^{8}$ T. Kafka, ${ }^{26}$ S. M. S. Kasahara, ${ }^{14}$ J. J. Kim, ${ }^{21}$ G. Koizumi ${ }^{8}$ S. Kopp ${ }^{25}$ M. Kordosky, ${ }^{28,13}$ D. J. Koskinen, ${ }^{13,15}$ Z. Krahn, ${ }^{14}$ A. Kreymer, ${ }^{8}$ K. Lang, ${ }^{25}$ J. Ling, ${ }^{21}$ P. J. Litchfield, ${ }^{14}$ R. P. Litchfield ${ }^{17}$ L. Loiacono, ${ }^{25}$ P. Lucas, ${ }^{8}$ J. Ma, ${ }^{25}$ W. A. Mann, ${ }^{26}$ M. L. Marshak, ${ }^{14}$ J. S. Marshall, ${ }^{6}$ N. Mayer, ${ }^{12}$ A. M. McGowan, ${ }^{1,14, \dagger}$ R. Mehdiyev, ${ }^{25}$ J. R. Meier, ${ }^{14}$ M. D. Messier, ${ }^{12}$ C. J. Metelko, ${ }^{19}$ D. G. Michael, ${ }^{5,}$ W. H. Miller, ${ }^{14}$ S. R. Mishra, ${ }^{21}$ J. Mitchell, ${ }^{6}$ C. D. Moore, ${ }^{8}$ J. Morfín, ${ }^{8}$ L. Mualem, ${ }^{5}$ S. Mufson, ${ }^{12}$ J. Musser, ${ }^{12}$ D. Naples, ${ }^{18}$ J. K. Nelson, ${ }^{28}$ H. B. Newman, ${ }^{5}$ R. J. Nichol, ${ }^{13}$ T. C. Nicholls, ${ }^{19}$ J. P. Ochoa-Ricoux,${ }^{5}$ W. P. Oliver, ${ }^{26}$ T. Osiecki, ${ }^{25}$ R. Ospanov, ${ }^{25,8}$ J. Paley, ${ }^{12}$ V. Paolone,${ }^{18}$ R. B. Patterson, ${ }^{5}$ Ž. Pavlović, ${ }^{25}$ G. Pawloski, ${ }^{22}$ G. F. Pearce, ${ }^{19}$ D. A. Petyt, ${ }^{14}$ R. Pittam, ${ }^{17}$ R. K. Plunkett, ${ }^{8}$ A. Rahaman, ${ }^{21}$ R. A. Rameika, ${ }^{8}$ T. M. Raufer, ${ }^{19,17}$ B. Rebel, ${ }^{8}$ P. A. Rodrigues, ${ }^{17}$ C. Rosenfeld, ${ }^{21}$ H. A. Rubin, ${ }^{11}$ V. A. Ryabov, ${ }^{32}$ M. C. Sanchez, ${ }^{1,9}$ N. Saoulidou, ${ }^{8}$ J. Schneps, ${ }^{26}$ P. Schreiner, ${ }^{3}$ V. K. Semenov, ${ }^{33}$ P. Shanahan, ${ }^{8}$ W. Smart, ${ }^{8}$ C. Smith,${ }^{13}$ A. Sousa,${ }^{9,17}$ P. Stamoulis, ${ }^{2}$ M. Strait,${ }^{14}$ N. Tagg, ${ }^{16,26}$ R. L. Talaga, ${ }^{1}$ J. Thomas, ${ }^{13}$ M. A. Thomson, ${ }^{6}$ G. Tinti, ${ }^{17}$ R. Toner, ${ }^{6}$ V. A. Tsarev, ${ }^{32}$ G. Tzanakos, ${ }^{2}$ J. Urheim,,${ }^{12}$ P. Vahle,${ }^{28,13}$ B. Viren, ${ }^{4}$ M. Watabe, ${ }^{24}$ A. Weber ${ }^{17}$ R. C. Webb,${ }^{24}$ N. West,${ }^{17}$ C. White,${ }^{11}$ L. Whitehead, ${ }^{4}$ S. G. Wojcicki, ${ }^{22}$ D. M. Wright,${ }^{29}$ T. Yang, ${ }^{22}$ M. Zois, ${ }^{2}$ K. Zhang, ${ }^{4}$ and R. Zwaska ${ }^{8}$

(MINOS Collaboration)

\footnotetext{
${ }^{1}$ Argonne National Laboratory, Argonne, Illinois 60439, USA

${ }^{2}$ Department of Physics, University of Athens, GR-15771 Athens, Greece

${ }^{3}$ Physics Department, Benedictine University, Lisle, Illinois 60532, USA

${ }^{4}$ Brookhaven National Laboratory, Upton, New York 11973, USA

${ }^{5}$ Lauritsen Laboratory, California Institute of Technology, Pasadena, California 91125, USA

${ }^{6}$ Cavendish Laboratory, University of Cambridge, Madingley Road, Cambridge CB3 OHE, United Kingdom

${ }^{7}$ Universidade Estadual de Campinas, IF-UNICAMP, CP 6165, 13083-970, Campinas, SP, Brazil

${ }^{8}$ Fermi National Accelerator Laboratory, Batavia, Illinois 60510, USA

${ }^{9}$ Department of Physics, Harvard University, Cambridge, Massachusetts 02138, USA

${ }^{10}$ Holy Cross College, Notre Dame, Indiana 46556, USA

${ }^{11}$ Physics Division, Illinois Institute of Technology, Chicago, Illinois 60616, USA

${ }^{12}$ Indiana University, Bloomington, Indiana 47405, USA

${ }^{13}$ Department of Physics and Astronomy, University College London, Gower Street, London WC1E 6BT, United Kingdom

${ }^{14}$ University of Minnesota, Minneapolis, Minnesota 55455, USA

${ }^{15}$ Department of Physics, University of Minnesota-Duluth, Duluth, Minnesota 55812, USA

${ }^{16}$ Otterbein College, Westerville, Ohio 43081, USA

${ }^{17}$ Subdepartment of Particle Physics, University of Oxford, Oxford OX1 3RH, United Kingdom

${ }^{18}$ Department of Physics and Astronomy, University of Pittsburgh, Pittsburgh, Pennsylvania 15260, USA

${ }^{19}$ Rutherford Appleton Laboratory, Science and Technology Facilities Council, OX11 OQX, United Kingdom

${ }^{20}$ Instituto de Física, Universidade de São Paulo, CP 66318, 05315-970, São Paulo, SP, Brazil

${ }^{21}$ Department of Physics and Astronomy, University of South Carolina, Columbia, South Carolina 29208, USA

${ }^{22}$ Department of Physics, Stanford University, Stanford, California 94305, USA

${ }^{23}$ Department of Physics and Astronomy, University of Sussex, Falmer, Brighton BN1 9QH, United Kingdom

${ }^{24}$ Physics Department, Texas A\&M University, College Station, Texas 77843, USA

${ }^{25}$ Department of Physics, University of Texas at Austin, 1 University Station C1600, Austin, Texas 78712, USA

${ }^{26}$ Physics Department, Tufts University, Medford, Massachusetts 02155, USA

${ }^{27}$ Department of Physics, University of Warsaw, Hoża 69, PL-00-681 Warsaw, Poland

${ }^{28}$ Department of Physics, College of William \& Mary, Williamsburg, Virginia 23187, USA

${ }^{29}$ Lawrence Livermore National Laboratory, Livermore, California 94550, USA

${ }^{30}$ Physics Department, Western Washington University, Bellingham, Washington 98225, USA
} 


\author{
${ }^{31}$ Physics Department, University of Wisconsin, Madison, Wisconsin 53706, USA \\ ${ }^{32}$ Nuclear Physics Department, Lebedev Physical Institute, Leninsky Prospect 53, 119991 Moscow, Russia \\ ${ }^{33}$ Institute for High Energy Physics, Protvino, Moscow Region RU-140284, Russia
}

(Received 12 October 2009; published 8 April 2010)

\begin{abstract}
The energy dependence of the neutrino-iron and antineutrino-iron inclusive charged-current cross sections and their ratio have been measured using a high-statistics sample with the MINOS near detector exposed to the NuMI beam from the main injector at Fermilab. Neutrino and antineutrino fluxes were determined using a low hadronic energy subsample of charged-current events. We report measurements of $\nu$-Fe $(\bar{\nu}-\mathrm{Fe})$ cross section in the energy range $3-50 \mathrm{GeV}(5-50 \mathrm{GeV})$ with precision of $2 \%-8 \%(3 \%-9 \%)$ and their ratio which is measured with precision $2 \%-8 \%$. The data set spans the region from low energy, where accurate measurements are sparse, up to the high-energy scaling region where the cross section is well understood.
\end{abstract}

DOI: 10.1103/PhysRevD.81.072002

PACS numbers: $13.15 .+\mathrm{g}$

\section{INTRODUCTION}

Neutrino-nucleon and antineutrino-nucleon chargedcurrent $\left(\nu_{\mu} N \mathrm{CC}\right.$ and $\left.\bar{\nu}_{\mu} N \mathrm{CC}\right)$ inclusive cross sections above $30 \mathrm{GeV}$ have been determined by several experiments [1-3] with a combined precision of 2\% [4]. The measured cross sections at these energies have a linear dependence on energy, which agrees well with the prediction of the quark parton model (QPM) [5].

At lower energies, the cross section is both less well measured and difficult to model due to overlapping contributions from quasielastic processes $\left(\nu_{\mu}+n \rightarrow\right.$ $\mu^{-}+p$ ), resonance excitation followed by subsequent decay, and the onset of deeply inelastic scattering (DIS). This energy range is of particular interest to ongoing and future neutrino oscillation searches in MINOS, $\mathrm{NO} \nu \mathrm{A}$ [6], and T2K [7]. Most cross section measurements in the $E_{\nu}<$ $30 \mathrm{GeV}$ range [8-14] have uncertainties of the order of $10 \%$. Recently, NOMAD [15] measured the cross section down to $2.5 \mathrm{GeV}$ with a precision of better than $4 \%$. However, this result relies on a particle production model tuned to data [16] to predict the neutrino flux. In this paper we present a measurement of the $\nu_{\mu} N \mathrm{CC}$ cross section with a precision from $2 \%-8 \%$, covering the $3-50 \mathrm{GeV}$ energy range using the MINOS near detector. Our analysis uses a low hadronic energy subsample to determine the flux shape $[17,18]$.

Antineutrino-nucleon charged-current cross sections in the $E_{\nu}<30 \mathrm{GeV}$ range suffer from the same complications listed above and tend to be even less well measured. Several experiments reported results $[11-13,19,20]$; however data coverage in energy was sparse and these measurements typically have larger than $10 \%$ uncertainty. Our

\footnotetext{
*Present address: Department of Physics and Astrophysics, University of Delhi, Delhi 110007, India.

${ }^{\dagger}$ Present address: Physics Department, St. John Fisher College, Rochester, NY 14618 USA.

ॠDeceased.

${ }^{\S}$ Present address: Physics and Astronomy, University of Pennsylvania, Philadelphia, PA 19104, USA.
}

measurement has higher precision, with uncertainties which range from $3 \%-9 \%$.

The $\bar{\nu}_{\mu} N$ CC to $\nu_{\mu} N$ CC cross section ratio, $r=$ $\sigma^{\bar{\nu}} / \sigma^{\nu}$, has been measured with a combined precision of better than $1 \%$ at high energies [17,21] but only one dedicated measurement [22] has been performed in the $E_{\nu}<30 \mathrm{GeV}$ range. Gargamelle [22] reports measurements of $r$ from $1-10 \mathrm{GeV}$ with precision of about $20 \%$. Our result substantially adds both coverage and precision to the determination of $r$. The ratio is more precisely determined than either cross section measured separately due to a partial cancellation of most systematic effects and a cancellation of the normalization uncertainty.

The results in this paper can be used to tune and improve neutrino interaction generator models $[23,24]$. For example, neutrino scattering data are required for the modeling of the axial vector contribution to the cross section [25]. Also, the cross section ratio $r$ is particularly sensitive to the modeling of $x F_{3}$, the parity violating structure function, which enters into the numerator and denominator with opposite sign, and to the antiquark content of the nucleon, which contributes differently to neutrino and antineutrino scattering. In addition, at $5 \mathrm{GeV}$, about $70 \%$ of our event sample has negative 4-momentum transfer squared, $Q^{2}$, of less than $1.5 \mathrm{GeV}^{2}$. This large, low- $Q^{2}$ sample provides model sensitivity to the low- $Q^{2}$ QCD contributions (higher order QCD, higher-twist, and target mass corrections) which are difficult to calculate.

\section{Overview of the analysis}

The $\nu_{\mu} \mathrm{CC}$ and $\bar{\nu}_{\mu} \mathrm{CC}$ total cross sections as a function of incoming neutrino energy $E$ are determined from the inclusive charged-current interaction rate and the incident neutrino flux. A sample of CC events ("cross section" sample) is selected and a subsample of these events with low hadronic energy ("flux" sample) is defined. A Monte Carlo simulation which includes detailed detector geometry and response is used to correct the flux and cross section samples for detector acceptance and smearing effects. 
Neutrino and antineutrino differential cross sections, $d \sigma^{\nu, \bar{\nu}} / d \nu$, approach the same constant value, independent of energy, in the limit of low- $\nu$, where $\nu$ is the energy transferred to the hadronic system. A method which exploits this feature is used to determine the energy dependence of the flux from the flux sample, which is then normalized using the world average cross section value measured above $30 \mathrm{GeV}$. To accomplish this we make use of the full range of our data sample, which overlaps with the high-energy measurements in the $30-50 \mathrm{GeV}$ region. This "low- $\nu$ " method has been used previously at high energies [17,18] and here it is adapted to the $E<30 \mathrm{GeV}$ range.

The neutrino beam, detector and the Monte Carlo simulation of the experiment are described in Sec. II. Section III describes the event sample selection and the methods for extracting the flux and the cross section. A discussion of systematic uncertainties and results are given in Secs. IV and $\mathrm{V}$, respectively.

\section{BEAM LINE AND DETECTOR}

MINOS is a two-detector, long baseline neutrino oscillation experiment using the NuMI (neutrinos at main injector) neutrino beam at Fermilab. The oscillation parameters are measured [26,27] by comparing the $\nu_{\mu}$ energy spectra at the near detector located at Fermilab and the far detector located $734 \mathrm{~km}$ away in the Soudan Mine in northern Minnesota. In this section we describe the neutrino beam, the near detector, and the Monte Carlo simulation. More detailed descriptions of the beam line and the MINOS detectors are given elsewhere [28].

\section{A. Neutrino beam}

The NuMI neutrino beam is produced from $120 \mathrm{GeV}$ protons extracted in a $10 \mu \mathrm{s}$ spill from the main injector which impinge on a graphite target, with a typical intensity for the data presented here of $2.2 \times 10^{13}$ protons on target (PoT) per spill. Charged particles produced in the target, mainly pions and kaons, are focused by a pair of toroidal magnets called horns into a $675 \mathrm{~m}$ long decay volume where the mesons decay to muons and neutrinos. The decay region is followed by a hadron absorber where remaining mesons and protons are stopped. The neutrino beam then traverses $240 \mathrm{~m}$ of unexcavated rock before reaching the near detector located $1.04 \mathrm{~km}$ from the target.

Data for this analysis were collected in "low-energy" beam mode in which the downstream end of the target is placed $10 \mathrm{~cm}$ from the neck of the first focusing horn and the current in the horns is $185 \mathrm{kA}$, with the polarity set to focus positively charged mesons. The Monte Carlo simulation predicts the composition of the event sample to be $92.9 \% \nu_{\mu}, 5.8 \% \bar{\nu}_{\mu}$, and $1.3 \% \nu_{e}+\bar{\nu}_{e}$. Figure 1 shows the simulated flux spectrum of the $\nu_{\mu}$ and $\bar{\nu}_{\mu}$ in the beam. The $\nu_{\mu}$ component of the beam, which results primarily from

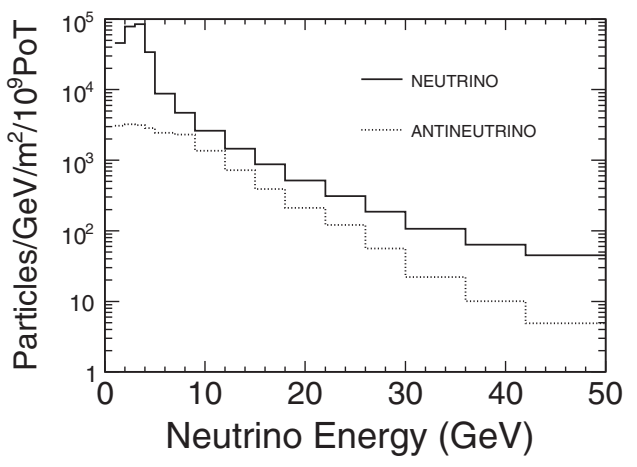

FIG. 1. The muon neutrino and antineutrino flux at the center of the near detector as calculated by the NuMI beam simulation.

focused $\pi^{+}$and $K^{+}$, peaks between 3 and $4 \mathrm{GeV}$ with a long tail. The $\bar{\nu}_{\mu}$ component arises mainly from low transverse momentum $\pi^{-}$and $K^{-}$traveling through the neck of both horns, where they undergo little defocusing. This results in a spectrum with no focusing peak and greater mean energy.

\section{B. Near detector}

The near detector is a tracking calorimeter composed of planes of magnetized iron and plastic scintillator. A toroidal magnetic field with an average strength of $1.3 \mathrm{~T}$ provides a measure of muon momentum from curvature and is used to distinguish $\nu_{\mu}$ and $\bar{\nu}_{\mu} \mathrm{CC}$ interactions based on the charge sign of the final state muon. In normal operational mode the field is set to focus negative muons.

The near detector, illustrated in Fig. 2, consists of 282 steel plates, $2.54 \mathrm{~cm}$ thick, of which 152 are instrumented with $1 \mathrm{~cm}$ thick scintillator planes. The scintillator planes are made of $4.1 \mathrm{~cm}$ wide strips oriented $\pm 45^{\circ}$ with respect to the vertical and alternating $\pm 90^{\circ}$ in successive planes. The strips are read out with wavelength shifting fibers connected to multianode photomultiplier tubes (PMT). Every fifth plane throughout the detector is fully instrumented with a scintillator layer. In the upstream calorimeter region, comprising the first 120 planes, each of the four intervening planes has partial scintillator coverage. The calorimeter region is used to measure energy deposited by neutrino-induced hadronic showers. Event vertices are required to be within a fiducial volume contained in the calorimeter. The downstream 162 planes of the detector form the muon spectrometer.

In the low-energy NuMI beam configuration, the typical interaction rate in the near detector is about 16 events in a $10 \mu$ s spill. Events are separated using timing and spatial information. The events accepted for this analysis were from interactions occurring during a $13 \mu$ s long gate synchronized to the beam spill. The readout electronics continuously digitize the PMT signals in 19 ns samples without dead time throughout the spill. In between beam spills, cosmic ray muon data are recorded with less than $1 \%$ dead time. 

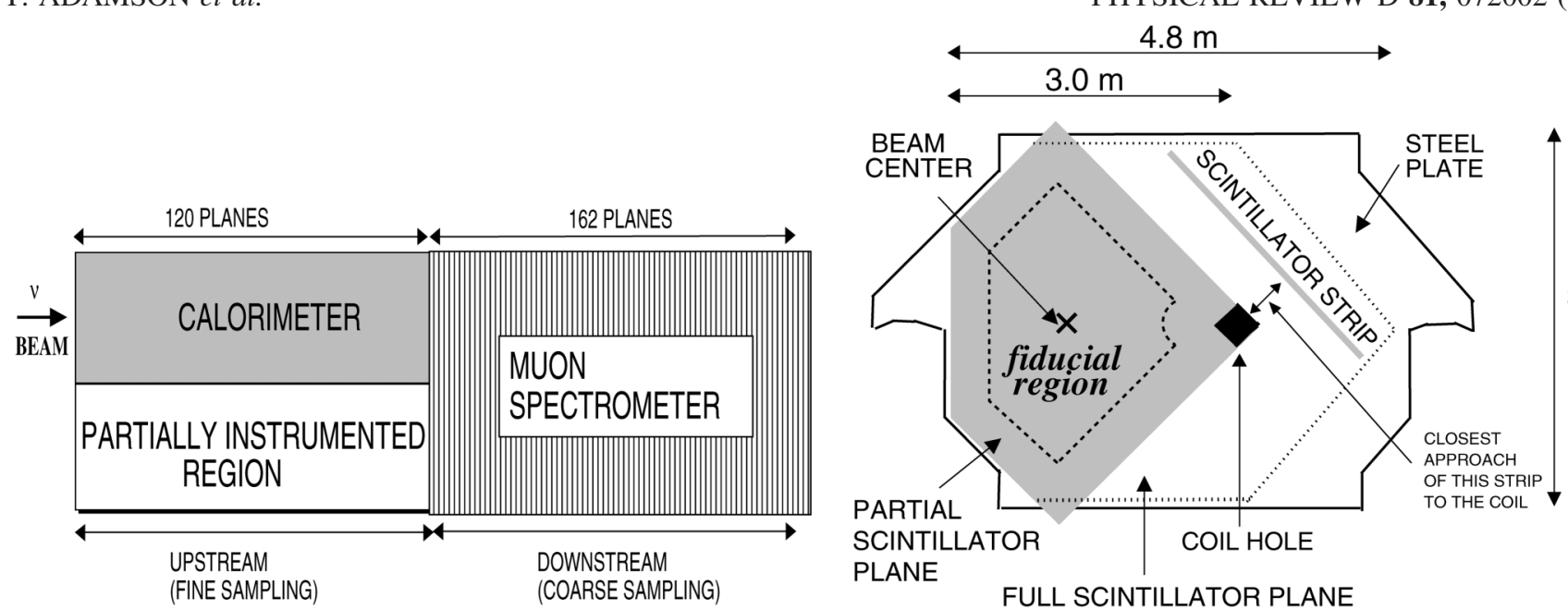

$3.8 \mathrm{~m}$

FIG. 2. Left panel: top view of the near detector, showing the calorimeter and muon spectrometer. The drawing is not to scale. Right panel: transverse view of a near detector plane. The shaded area shows a partially instrumented active scintillator plane and the dashed line within shows the boundary of the fiducial region. The dotted line shows the outline of a fully instrumented scintillator plane.

The detector is calibrated in several steps that convert the raw PMT signal to deposited energy [28]. The nonlinearity of the electronics is measured with charge injection; relative PMT gains are measured with an in situ light injection system; variations in the light output between scintillator strips and along the strips are corrected with cosmic ray muons and a radioactive source scanner. Cosmic ray muons which stop in the detector are used to calibrate the measured signal to energy lost by muons passing through the scintillator strips. The detector simulation is tuned to emulate the actual detector response at all stages in the calibration chain.

\section{Beam and detector Monte Carlo simulation}

A Monte Carlo simulation is used to model the production of the neutrino beam, interaction of neutrinos in and around the detector, and the detector response, which is simulated using GEANT3 [29]. The beam model includes a simulation of secondary hadron production from proton interactions [30] and the propagation of these hadrons. Their reinteraction and decay products are also tracked through the target, magnetic horns, and decay region. This simulation produces an initial estimate of the flux, which is later replaced by the flux extracted using the method described below.

Neutrino interactions in the detector are simulated using the NEUGEN3 [23] event generator. The simulation of quasielastic interactions, which dominate at low energies, is based on the Llewellyn-Smith [31] model, while intermediate-energy resonance interactions are simulated according to the Rein-Sehgal model [32,33]. Both models assume a dipole parametrization of the axial part of the cross section that depends on the axial mass parameters $M_{A}(Q E L)$ and $M_{A}(R E S)$, taken to be $0.99 \pm 0.15$ and $1.12 \pm 0.17 \mathrm{GeV}$, respectively. A transition is made be- tween resonance production and the DIS model by phasing out the former and phasing in the latter over the hadronic invariant mass range, $1.7<W<2.0 \mathrm{GeV}$. The sum of the resonance and DIS contributions are constrained to match total cross section data.

DIS interactions, which dominate at high energy, are based on an effective leading order model by Bodek et al. [34]. The Bjorken scaling variable $x$ is replaced by an effective scaling variable that depends on two parameters $A_{h t}$ and $B_{h t}$, where $A_{h t}$ accounts for target mass effects and higher-twist terms. $B_{h t}$ depends on the transverse momentum of the initial state quark. The model is fit to charged lepton scattering data [34] and gives the parameters $A_{h t}$ and $B_{h t}$ and correction factors $\left(C_{v 1 u}, C_{v 2 u}, C_{v 1 d}, C_{v 2 d}, C_{s 1 d}\right.$, and $C_{s 1 u}$ ) for valence and sea up and down quark parton distribution functions. The uncertainties on these parameters were not readily available so a study was performed to estimate them and their effect on this cross section measurement (see Sec. IV).

The cross section in the transition region from resonance to DIS is expressed as a sum of a pure-resonance cross section and a nonresonance contribution from DIS. The sum is tuned to describe low multiplicity final state data in this region [23]. For DIS interactions, the final state hadronic system is modeled with KNO scaling [35], which transitions to PYTHIA/JETSET [36] at hadronic invariant mass $W=3 \mathrm{GeV}$. The total neutrino cross section is tuned by a scale factor so that the cross section at $100 \mathrm{GeV}$ matches the world average of measurements.

The dynamics of hadron formation in the target nucleus and reinteraction of hadrons after formation modify the visible hadronic shower energy. These effects are simulated using a cascade Monte Carlo anchored to $\pi N, p N$ and $\pi \mathrm{Fe}$ and $p \mathrm{Fe}$ scattering data and validated against neutrinodeuterium and neutrino-neon scattering data [37,38]. A treatment of hadron formation time is included [39]. 


\section{ANALYSIS}

The CC total cross sections are measured from the inclusive CC scattering rate, $\Gamma_{\mathrm{CC}}^{\nu(\bar{\nu})}(E)$, and the incident neutrino flux, $\Phi^{\nu(\bar{\nu})}(E)$. A sample of CC events, $N_{\mathrm{CC}}^{\nu(\bar{\nu})}(E)$, is selected and then corrected for acceptance and backgrounds to determine $\Gamma_{\mathrm{CC}}^{\nu(\bar{\nu})}(E)$. A flux sample, $F^{\nu(\bar{\nu})}(E)$, consisting of the subset of $N_{\mathrm{CC}}^{\nu(\bar{\nu})}(E)$ with low $\nu$ (in the lab frame $\nu=E_{\text {had }}$, the energy measured at the hadronic vertex), is also defined and corrected for acceptance, backgrounds, and for a small energy dependence using our Monte Carlo model to yield $\Phi^{\nu(\bar{\nu})}(E)$. The event reconstruction and selection of these samples to form the cross section are described in this section.

The data used in this analysis were collected between June 2005 and April 2007 and correspond to an exposure of $2.45 \times 10^{20}$ PoT. The MC sample is almost double the data, corresponding to $4.4 \times 10^{20}$ PoT.

\section{A. Event reconstruction}

Neutrino events are identified using the timing and spatial pattern of energy deposited in the scintillator strips. Muon tracks are recognized as a string of hit strips typically spanning more than 10 steel plates. For muons that stop in the detector the energy is computed from range according to the energy loss tables of Groom et al. [40]. A systematic uncertainty of $2 \%$ is assigned to the energy measured from range, arising from uncertainties in the range tables, the variation in material composition, and the accuracy of our track length reconstruction. The momentum of muons exiting the detector is measured using the curvature of their trajectory in the detector's magnetic field. A 4\% systematic uncertainty is assigned to our knowledge of the absolute muon momentum measurement from curvature. This is assessed by comparing the energy measured with curvature to the independent measurement from range using tracks that stop in the detector, and by folding in underlying uncertainties in the detector's magnetic field [41]. The resolution for muon momentum measured from range is $5 \%$ while that measured from curvature has non-Gaussian tails and width of approximately $10 \%$.

The vertex of a neutrino interaction is taken to be at the start of a reconstructed track. Hit strips near the vertex which are not included in the track are identified as coming from hadrons produced in the interaction. Their summed signal is converted to energy using a lookup table derived from simulated showers to form the hadronic shower energy, $E_{\text {had }}$. The response of our detector to single hadrons was measured in an exposure of a smaller version of the detector to a test beam [42]. The measured test beam detector response was used to tune our simulations. The absolute energy scale of the detector's response to hadronic particles is modeled to an accuracy of 5.6\% [27,43], which we take as the hadronic energy scale uncertainty in the cross section measurement (see Sec. IV).

\section{B. CC Event selection}

The inclusive charged-current sample $N_{\mathrm{CC}}^{\nu(\bar{\nu})}(E)$ is selected using the following criteria:

(1) Fiducial volume: Selected events have a vertex position along the detector axis between 0.5 and $4.0 \mathrm{~m}$, measured from the upstream face of the detector. In the plane transverse to the detector axis, the vertex is required to be more than $0.5 \mathrm{~m}$ from the edge of an active scintillator plane and outside of a $0.8 \mathrm{~m}$ radius centered at the coil hole. The outline of the fiducial region is shown in Fig. 2.

(2) Coil hole: The coil hole is uninstrumented and variations in the material composition and magnetic field are somewhat larger in the region around it. To reduce the effect of these uncertainties, events with tracks that spend a significant fraction of their path length near the hole are removed from the event sample. A minimum of $95 \%$ of hit strips in the event is required to be farther than $0.3 \mathrm{~m}$ from the center at closest approach (see Fig. 2).

(3) Track energy: The energy of the muon must be greater than $1.5 \mathrm{GeV}$. This requirement rejects neutral-current (NC) background events, which populate the low-energy region, and short, poorly reconstructed tracks.

(4) Track quality: The track fitting procedure yields a measurement of the muon momentum with an associated uncertainty. The track fit is required to be convergent and have an uncertainty of less than $30 \%$. In addition, we require the track's longitudinal start positions in each view to be less than six planes apart.

(5) Neutrino energy: The reconstructed neutrino energy, $E$, which is the sum of the track and shower energies, is required to be greater than $3 \mathrm{GeV}$ $(5 \mathrm{GeV})$ for the neutrino (antineutrino) sample and less than $50 \mathrm{GeV}$. The minimum energy requirements are imposed to minimize the overlap of the inclusive CC sample and the flux sample, which is substantial below these values. Above the maximum energy cut, resolution of the track momentum measurement from curvature degrades as the tracks become straighter.

The event sample is divided into two categories depending on whether the track stops in or exits the detector. For exiting events, the muon leaves the detector through the back or side, or passes into the uninstrumented coil hole region. The stopping and exiting samples are further differentiated based on whether they end in the upstream or downstream region (see Fig. 2) because of the difference in sampling in the two regions.

The $\bar{\nu}_{\mu}$ CC sample is selected by requiring the sign of the track curvature measurement to be positive. This sample has a higher fractional contamination from wrong-sign events (misidentified $\mu^{-}$tracks) due to the much larger $\nu_{\mu}$ 
component of the beam. The following additional requirements are imposed to the $\bar{\nu}_{\mu} \mathrm{CC}$ sample to reduce this contamination:

(1) Bend away from coil: We require that the track bend away from the magnet coil hole to reject positive charge track candidates whose curvature is mismeasured by the tracker. An angle is defined in the transverse plane by forming a straight line from the extrapolated track end point in absence of a magnetic field to the observed track end point, and a line from the magnet coil hole center to the observed interaction point [41]. For a particle bending toward the coil, this angle will be near $\pi \mathrm{rad}$, while for a defocused $\mu^{+}$it will be near 0 or $2 \pi$ rad. For the antineutrino sample, we select a value for this angle less than 1.04 rad or greater than 5.24 rad.

(2) Number of hit planes: We keep events in which the difference in the number of hit planes along the track between the two views is less than five. Events which are rejected by this cut usually enter the uninstrumented region in one view yielding an unreliable determination of the charge sign.

Downstream exiting tracks: Only events with tracks that exit the detector in the downstream region are used for the antineutrino analysis. The rejected samples have high contamination from $\mathrm{NC}$ and misidentified $\nu_{\mu}$ CC (wrong-sign) events.

Table I shows the effect of the selection criteria on the neutrino and the antineutrino reconstructed samples. The minimum track energy cut has the largest effect, resulting in an approximately $30 \%$ loss in the $\nu_{\mu}$ sample and $60 \%$ in the $\bar{\nu}_{\mu}$ sample. The cut removes primarily NC events, which arise from both neutrinos and antineutrinos, and therefore affects the smaller $\bar{\nu}_{\mu}$ sample more. The track quality cut also has a larger effect on the $\bar{\nu}_{\mu}$ sample. A large fraction of the $\nu_{\mu} \mathrm{CC}$ tracks whose charge has been mismeasured due to poor curvature determination is removed from the $\bar{\nu}_{\mu}$ sample by this cut. After all selections have been applied, the inclusive event sample $N_{\mathrm{CC}}^{\nu(\bar{\nu})}(E)$ consists of $1.94 \times 10^{6} \nu_{\mu}$ and $1.59 \times 10^{5} \bar{\nu}_{\mu}$ events.

The CC sample is organized into energy bins and corrected for detector acceptance $A_{\mathrm{CC}}^{\nu(\bar{\nu})}(E)$ and backgrounds

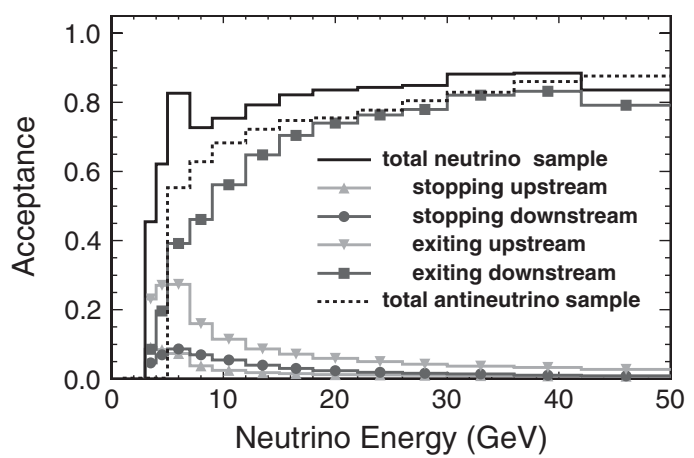

FIG. 3. The detector acceptance $A_{\mathrm{CC}}^{\nu(\bar{\nu})}(E)$ for the inclusive charged-current $\nu_{\mu}$ and $\bar{\nu}_{\mu}$ samples. Only exiting downstream events are used in the $\bar{\nu}_{\mu}$ analysis.

$B_{\mathrm{CC}}^{\nu(\bar{\nu})}(E)$ to obtain the $\mathrm{CC}$ scattering rate, $\Gamma_{\mathrm{CC}}^{\nu(\bar{\nu})}(E)=$ $\left(N_{\mathrm{CC}}^{\nu(\bar{\nu})}(E)-B_{\mathrm{CC}}^{\nu(\bar{\nu})}(E)\right) / A_{\mathrm{CC}}^{\nu(\bar{\nu})}(E)$, where $A_{\mathrm{CC}}^{\nu(\bar{\nu})}(E)$, shown in Fig. 3, represents the number of Monte Carlo events reconstructed in a given bin divided by the number generated in that bin. The decrease in acceptance at low energy is due to the minimum muon energy requirement. For neutrinos, the shape below $10 \mathrm{GeV}$ is determined by the geometry of the detector and overlap of the stopping and exiting samples, which have different resolutions. The contributions from each subsample are also shown in Fig. 3.

We use our simulation to estimate the backgrounds from $\mathrm{NC}$ and wrong-sign events. As shown in Fig. 4, the NC background is less than $2 \%$ for both neutrinos and antineutrinos. It increases with energy due to the contribution from high-inelasticity events in which the primary track is misidentified or perturbed by hits from the hadronic shower particles. The wrong-sign contamination is negligible in the neutrino sample but sizable in the antineutrino sample, up to 5\% at high energy. Wrong-sign background events at low energy come from $\nu_{\mu} \mathrm{CC}$ events in the peak of the neutrino beam, while at higher energies, the throughgoing muons have increasingly larger bend radii, making their charge determination less certain.

\section{Flux extraction}

The low- $\nu$ method $[17,18]$ relies on the independence of the differential cross section, $d \sigma^{\nu, \bar{\nu}} / d \nu$, with energy in the

TABLE I. Effect of the selection criteria on the negative (left) and positive (right) charge reconstructed track samples. Each row shows the number of events remaining after each successive cut. The numbers in parentheses show the percentage of events removed by each cut compared with the previous row.

\begin{tabular}{lcc}
\hline \hline Selection criterion & Track charge $<0(\%$ removed $)$ & Track charge $>0(\%$ removed $)$ \\
\hline Track vertex in fiducial volume & 3608572 & 841986 \\
$E_{\mu}>1.5 \mathrm{GeV}$ & $2571917(28.7 \%)$ & $344110(59.1 \%)$ \\
Track quality cut & $2351328(8.6 \%)$ & $282657(17.8 \%)$ \\
$3<E_{\nu}<50 \mathrm{GeV}$ & $1941019(17.5 \%)$ & $235024(16.9 \%)$ \\
$(5<E<50 \mathrm{GeV}$ for track charge $>0)$ & $\ldots$ & $159880(32 \%)$ \\
Additional $\bar{\nu}_{\mu}$ cuts & $\ldots$ & $(32 \%)$ \\
\hline \hline
\end{tabular}



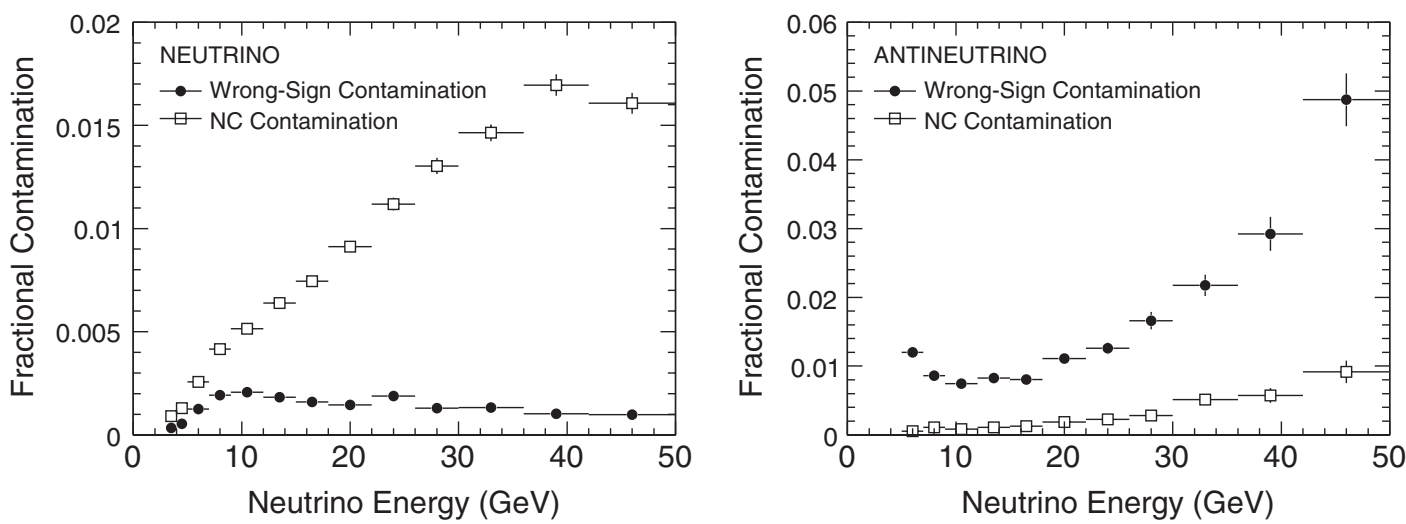

FIG. 4. Neutral-current and wrong-sign backgrounds in the neutrino (left panel) and antineutrino (right panel) selected chargedcurrent samples as calculated from the Monte Carlo event sample. The error bars show the statistical uncertainty only.

limit $\nu \rightarrow 0$. The differential dependence of the neutrino (antineutrino) cross section, $d^{2} \sigma^{\nu, \bar{\nu}} / d x d y$, on inelasticity, $y=\nu / E$, and the Bjorken scaling variable, $x$, can be written as

$$
\begin{aligned}
\frac{d^{2} \sigma^{\nu(\bar{\nu})}}{d x d y}= & \frac{G_{F}^{2} M E}{\pi}\left(\left[1-y\left(1+\frac{M x}{2 E}\right)+\frac{y^{2}}{2}\right.\right. \\
& \left.\left.\times\left(\frac{1+\left(\frac{2 M x}{Q}\right)^{2}}{1+R_{L}}\right)\right] F_{2} \pm\left[y-\frac{y^{2}}{2}\right] x F_{3}\right),
\end{aligned}
$$

where $G_{F}$ is the Fermi weak coupling constant, $M$ is the proton mass, and $E$ is the incident neutrino energy. The plus sign in front of the $x F_{3}$ term is for neutrinos and the minus is for antineutrinos. The structure functions $F_{2}\left(x, Q^{2}\right), x F_{3}\left(x, Q^{2}\right)$, and $R_{L}\left(x, Q^{2}\right)$ depend on $x$ and $Q^{2}$, and $R_{L}$ is the ratio of the cross section for scattering from longitudinally to transversely polarized $W$ bosons. For quasielastic interactions the cross section can be written in this form with combinations of form factors replacing the structure functions.

Integrating over $x$, the differential dependence on $\nu$ can be written in the simplified form

$$
\frac{d \sigma^{\nu, \bar{\nu}}}{d \nu}=A\left(1+\frac{B}{A} \frac{\nu}{E}-\frac{C}{A} \frac{\nu^{2}}{2 E^{2}}\right) .
$$

The coefficients $A, B$, and $C$ depend on integrals over structure functions,

$$
\begin{aligned}
& A=\frac{G_{F}^{2} M}{\pi} \int F_{2}(x) d x, \\
& B=-\frac{G_{F}^{2} M}{\pi} \int\left(F_{2}(x) \mp x F_{3}(x)\right) d x, \\
& C=B-\frac{G_{F}^{2} M}{\pi} \int F_{2}(x) \tilde{R} d x,
\end{aligned}
$$

where

$$
\tilde{R}=\left(\frac{1+\frac{2 M x}{\nu}}{1+R_{L}}-\frac{M x}{\nu}-1\right)
$$

The factor $A$ is nearly the same for neutrino and antineutrino probes, ${ }^{1}$ however, the magnitude of the coefficient $B$ is larger for antineutrinos, where the $x F_{3}$ contribution is added, compared with the neutrino case where the term is subtracted. As discussed later, this makes the energy dependence correction needed in this method larger for the antineutrino flux shape. The $C$ term, which depends on $R_{L}$, is small.

For small $\nu / E$, Eq. (2) shows that the differential cross section becomes independent of energy and is equal to the same constant, $A$, for neutrinos and antineutrinos. Multiplying both sides by the flux, $\phi(E)$, and taking the limit $\nu \rightarrow 0$ gives

$$
\left.\frac{d N}{d \nu}\right|_{\nu \rightarrow 0}=A \Phi(E)
$$

Therefore, the flux in a given energy bin can be approximated using the number of events at low $\nu$.

We account for the small $\nu / E$ and $(\nu / E)^{2}$ dependence resulting from a finite $\nu_{0}$ in Eq. (2) using a low- $\nu$ correction

$$
S^{\nu(\bar{\nu})}\left(\nu_{0}, E\right)=\frac{\sigma\left(\nu<\nu_{0}, E\right)}{\sigma\left(\nu<\nu_{0}, E \rightarrow \infty\right)}
$$

that is calculated from our cross section model. The term $\sigma\left(\nu<\nu_{0}, E\right)$ is the value of the integrated cross section below our chosen $\nu_{0}$ cut at energy $E$, and $\sigma\left(\nu<\nu_{0}, E \rightarrow\right.$ $\infty)$ is its value in the high-energy limit.

This correction is applied to our selected flux sample, $F^{\nu(\bar{\nu})}(E)$, consisting of the subset of $N_{\text {CC }}^{\nu(\bar{\nu})}(E)$ with $\nu<\nu_{0}$ that is subsequently corrected for acceptance, $A_{\Phi}^{\nu(\bar{\nu})}(E)$, and backgrounds, $B_{\Phi}^{\nu(\bar{\nu})}(E)$,

\footnotetext{
${ }^{1}$ For an isoscalar target with only $u$ and $d$ quarks, $F_{2}^{\nu}=F_{2}^{\bar{\nu}}$, assuming isospin symmetry. Including $s$ quarks and CabibboKobayashi-Maskawa mixing gives a small difference term $F_{2}^{\nu}-$ $F_{2}^{\bar{\nu}}=-\frac{1}{2} V_{u s}^{2}\left(u_{v}+d_{v}\right)$, where $u_{v}$ and $d_{v}$ are the valence quark distributions. We apply a correction to account for this term to the antineutrino normalization.
} 


$$
\phi^{\nu(\bar{\nu})}(E)=\frac{F^{\nu(\bar{\nu})}(E)-B_{\Phi}^{\nu(\bar{\nu})}(E)}{S^{\nu(\bar{\nu})}\left(\nu_{0}, E\right) \times A_{\Phi}^{\nu(\bar{\nu})}(E)} .
$$

This yields the shape of the flux with energy. A normalization factor $H^{\nu}$, determined using external data, must be applied to give the absolute flux, $\Phi^{\nu(\bar{\nu})}(E)=H^{\nu} \phi^{\nu(\bar{\nu})}(E)$, as described in the next section.

Our choice of $\nu_{0}$ trades statistical precision for modeling uncertainty in determining $S^{\nu(\bar{\nu})}\left(\nu_{0}, E\right)$. To improve the statistical precision, we increase $\nu_{0}$ with energy while keeping the ratio $\nu / E$ and the resulting model dependence small. We set $\nu_{0}=1 \mathrm{GeV}$ for events with $E_{\nu}<9 \mathrm{GeV}, \nu_{0}=$ $2 \mathrm{GeV}$ for $9<E_{\nu}<18 \mathrm{GeV}$, and $\nu_{0}=5 \mathrm{GeV}$ for $E_{\nu}>$ $18 \mathrm{GeV}$. Figure 5 shows the size of the low- $\nu$ correction for neutrino and antineutrino samples. The correction for neutrinos is about $3 \%$ at $3 \mathrm{GeV}$ and for antineutrino is about $20 \%$ at $5 \mathrm{GeV}$.

The stronger inelasticity dependence of the antineutrino cross section results in the much larger correction for antineutrinos. In addition, antineutrino CC interactions have lower inelasticity on average, which causes a large overlap between the cross section and the flux samples. The overlap decreases with energy from $90 \%$ at $3 \mathrm{GeV}$ to about $60 \%$ at $6 \mathrm{GeV}$ for antineutrinos, whereas for neutrinos it is $60 \%$ at $3 \mathrm{GeV}$ and below $30 \%$ above $6 \mathrm{GeV}$ [44]. We therefore restrict our analysis to the region above $5 \mathrm{GeV}$ for the antineutrino sample.

The low- $\nu$ correction introduces a model dependence and model uncertainty to the flux determination. We account for this uncertainty in the flux by varying the model parameters described in Sec. IIC and recalculating the flux. The change in the correction when the model is varied is $1 \%$ or less because it is a fractional term in which the numerator and denominator are similarly affected.

Figure 6 and Table II show the extracted neutrino and antineutrino fluxes in the selected fiducial volume after

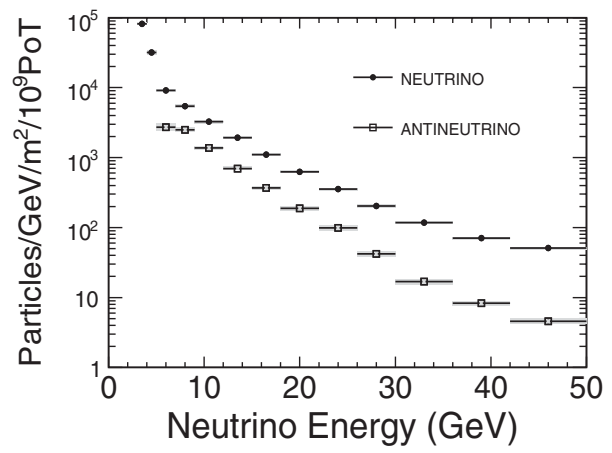

FIG. 6. Extracted flux as a function of neutrino energy. The error bars are too small to see on this log-scale plot, but are given in Table II.

normalization. The systematic uncertainties on the extracted flux are discussed in Sec. IV. We correct the input flux model shown in Fig. 1 by reweighting the simulation with the ratio of the extracted flux to the original simulated flux. The resulting corrections to the initial simulated flux are consistent with those obtained by a different technique [27] used for the MINOS oscillation analyses.

Figure 7 shows a comparison of the CC data sample and Monte Carlo simulation before and after flux reweighting is applied for the measured kinematic variables: the muon energy, hadronic shower energy, and muon track angle with respect to the beam direction. The agreement between Monte Carlo and data in all three distributions is significantly improved after the flux reweighting has been applied.

\section{Cross section extraction}

The energy dependence of the cross section is extracted by dividing the selected $\mathrm{CC}$ rate $\Gamma_{\mathrm{CC}}^{\nu(\bar{\nu})}(E)$ by the measured flux $\Phi^{\nu(\bar{\nu})}(E)$ in each energy bin. Explicitly including the corrections described above, the cross section is

$$
\frac{\sigma_{\mathrm{CC}}^{\nu(\bar{\nu})}(E)}{E}=\frac{1}{E} \frac{\Gamma_{\mathrm{CC}}^{\nu(\bar{\nu})}(E)}{\Phi^{\nu(\bar{\nu})}(E)}=\frac{1}{E}\left(\frac{\left(N_{\mathrm{CC}}^{\nu(\bar{\nu})}(E)-B_{\mathrm{CC}}^{\nu(\bar{\nu})}(E)\right) / A_{\mathrm{CC}}^{\nu(\bar{\nu})}(E)}{S^{\nu(\bar{\nu})}\left(\nu_{0}, E\right) \times H^{\nu} \times\left(F^{\nu(\bar{\nu})}(E)-B_{\Phi}^{\nu(\bar{\nu})}(E)\right) / A_{\Phi}^{\nu(\bar{\nu})}(E)}\right) .
$$
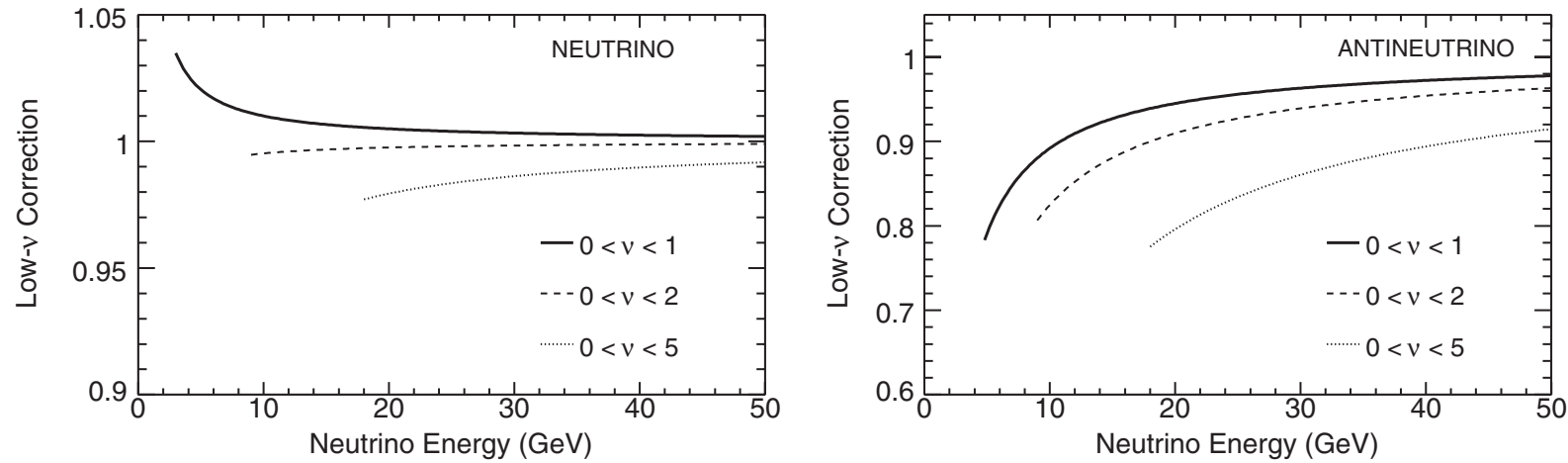

FIG. 5. The low- $\nu$ correction, $S^{\nu(\bar{\nu})}\left(\nu_{0}, E\right)$, applied to the flux sample for neutrinos (left panel) and antineutrinos (right panel). The solid lines show $S^{\nu(\bar{\nu})}\left(\nu_{0}, E\right)$ for $\nu<1 \mathrm{GeV}$ applied from 3-9 GeV (5-9 GeV for $\bar{\nu}$ ), dashed lines for $\nu<2 \mathrm{GeV}$ applied from 9$18 \mathrm{GeV}$, and the dotted lines for $\nu<5 \mathrm{GeV}$ applied above $18 \mathrm{GeV}$. 
TABLE II. Measured flux as a function of neutrino energy. Statistical, systematic, and normalization uncertainties are included in the error estimate.

\begin{tabular}{lcccc}
\hline \hline $\begin{array}{l}E \text { bin } \\
(\mathrm{GeV})\end{array}$ & $\nu$ flux & $\begin{array}{c}\text { Error } \\
\text { Particles } / \mathrm{GeV} / \mathrm{m}^{2} / 109 \text { PoT }\end{array}$ & $\begin{array}{c}\bar{\nu} \text { flux } \\
\text { Error }\end{array}$ \\
\hline $3-4$ & $8.05 \times 10^{4}$ & $5.2 \times 10^{3}$ & $\ldots$ & $\ldots$ \\
$4-5$ & $3.06 \times 10^{4}$ & $2.4 \times 10^{3}$ & $\ldots$ & $\ldots$ \\
$5-7$ & $9.07 \times 10^{3}$ & $5.3 \times 10^{2}$ & $2.80 \times 10^{3}$ & 330 \\
$7-9$ & $5.18 \times 10^{3}$ & $3.5 \times 10^{2}$ & $2.32 \times 10^{3}$ & 170 \\
$9-12$ & $3.21 \times 10^{3}$ & $2.2 \times 10^{2}$ & $1.32 \times 10^{3}$ & 85 \\
$12-15$ & $1.94 \times 10^{3}$ & $1.0 \times 10^{2}$ & $6.89 \times 10^{2}$ & 42 \\
$15-18$ & $1.09 \times 10^{3}$ & 65 & $3.79 \times 10^{2}$ & 24 \\
$18-22$ & 629 & 37 & 190 & 14 \\
$22-26$ & 348 & 20 & 86.3 & 7.8 \\
$26-30$ & 200 & 13 & 40.1 & 3.9 \\
$30-36$ & 119 & 6.8 & 19.3 & 1.9 \\
$36-42$ & 72.2 & 3.9 & 9.6 & 0.9 \\
$42-50$ & 51.6 & 2.8 & 4.9 & 0.5 \\
\hline \hline
\end{tabular}

Because our data extend above $30 \mathrm{GeV}$ we can take advantage of existing high-energy precision measurements $[1-3,11,19]$ to determine the flux normalization constant $H^{\nu} . H^{\nu}$ is chosen so that our measured $\nu_{\mu}$ flux results in an average $\nu_{\mu} \mathrm{CC}$ cross section value from $30-50 \mathrm{GeV}$ that agrees with the world average on an isoscalar target $(0.675 \pm 0.009) \times 10^{-38} \mathrm{~cm}^{2} / \mathrm{GeV}$ in the same energy range. The uncertainty on the normalization constant from the statistical precision of our data and that from the world average cross section are added in quadrature and applied as an uncertainty on our measured cross section at all energies. We apply a normalization correction to the antineutrino sample to account for the small difference in $F_{2}^{\nu}$ and $F_{2}^{\bar{\nu}}$. The correction, which is computed from our cross section model, is $1 \%$ for $\nu<1 \mathrm{GeV}, 2.6 \%$ for $\nu<$ $2 \mathrm{GeV}$, and $3.8 \%$ for the $\nu<5 \mathrm{GeV}$ sample [44].

After extracting the flux with the low- $\nu$ method, the cross section analysis is then repeated with the measured flux as input. This removes the effect of inaccuracies in the initial simulated flux on the acceptance corrections that are applied to both the flux and the cross section samples. The change in cross section between the final value and that extracted with the default simulated flux is less than $0.5 \%$ averaged over all data points. Since this effect is small we do not iterate the procedure further.

The cross section we report is that expected for an isoscalar target. The MINOS iron-scintillator detector has a $6.1 \%$ excess of neutrons over protons for which we correct using the NEUGEN3 cross section model [23]. The energy dependent corrections are about $-2 \%$ for neutrinos and $+2 \%$ for antineutrinos. We also apply corrections for radiative effects [45], which have an effect on the result of less than $1 \%$ at all energies.

The Appendix provides the measured raw ratio of cross section to flux data samples where both numerator and denominator are corrected only for detector effects and backgrounds. As described further therein, this allows one to remove the model dependence in the cross section extraction and to use an alternative cross section model to correct the ratio and compute cross sections.

\section{SYSTEMATIC UNCERTAINTIES}

Systematic uncertainties in the measurement from the following sources were considered: reconstructed muon and hadron energy scales, the effect of final state interactions on the measured energy, NC contamination, wrongsign contamination (antineutrino sample only), our lack of perfect understanding of the detector and event reconstruction, and cross section modeling. Each systematic uncertainty is evaluated independently and propagated through the analysis, including a recalculation of the absolute normalization of the result. Many systematic effects cause changes that are similar in the cross section and flux samples and therefore partially cancel in the measured cross section.

The largest uncertainty comes from knowledge of the absolute muon and hadronic energy scales discussed in Sec. II B. The muon energy scale is more important for the flux sample than for the cross section sample because a larger fraction of the neutrino energy per event is carried by the muon in the former. Conversely, the hadronic energy scale is less important in the flux sample.

Figure 8 shows the effects of muon and hadronic energy scale uncertainties on the extracted cross section. These are evaluated by applying the $1 \sigma$ shift in each scale factor to the data, extracting a new flux, and determining a new cross section, including a new normalization to the world data between 30 to $50 \mathrm{GeV}$. The resulting curves in Fig. 8 represent the change from the baseline cross section measurement. These uncertainties peak in the low-energy region and become small at high energies where the normalization is pinned.

The energy dependence of these uncertainties has a nontrivial shape because of the interplay of the shape of the flux spectrum and the method of normalizing to the world high-energy measurements. In particular, applying a muon energy scale factor shifts the observed flux in one direction and causes inflection points in the shape of the extracted flux near 6 and $14 \mathrm{GeV}$ for neutrinos, which propagate (with some cancellation) to the cross section analysis as shown in Fig. 8. A similar effect arises more directly in the cross section sample for the hadron energy scale. We show the $+1 \sigma$ systematic alone so that the shape distortion is clear. The $-1 \sigma$ distortion is the same shape but inverted in sign. The antineutrino analysis has fewer inflections because the spectrum is not peaked.

As described in Sec. II C, final state interactions affect the measured hadronic energy and are included in our Monte Carlo simulation. The uncertainty in their modeling contributes an effective hadronic energy scale uncertainty 

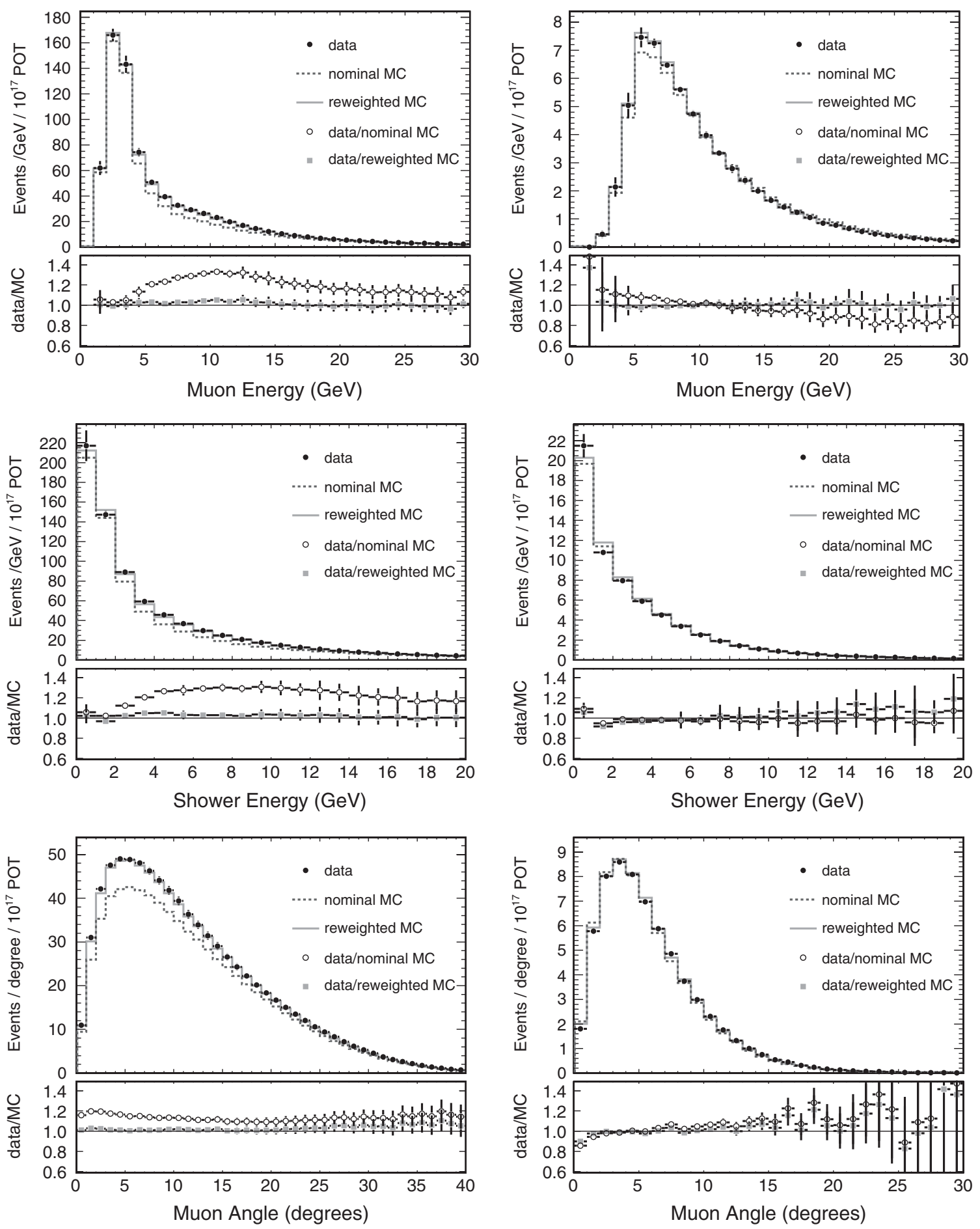

FIG. 7. Comparison of data and Monte Carlo simulation distributions for the kinematic variables $E_{\mu}, E_{\text {had }}$, and $\theta_{\mu}$ for neutrinos (left panels) and antineutrinos (right panels). The points show the data, the dashed lines show the nominal Monte Carlo model, and the solid lines show the Monte Carlo model after applying flux reweighting. The ratio of the data to the Monte Carlo simulation is shown below each distribution. The error bars show the statistical and systematic uncertainties added in quadrature.

of $8 \%$ below hadronic energy of $1 \mathrm{GeV}$, decreasing to $4 \%$ above $5 \mathrm{GeV}$. Their effect on the cross section, shown in Fig. 8, peaks at low energy and is fractionally larger for antineutrinos, which have a larger fraction of low hadron energy events due to their inelasticity distribution.
The uncertainty from our knowledge of the NC contamination is obtained by varying the value of the minimum $E_{\mu}$ requirement, which selects the $\mathrm{CC}$ sample, from its nominal value of 1.5 up to $2.0 \mathrm{GeV}$ and down to $1.0 \mathrm{GeV}$. The resulting change in $\sigma / E$ is small and corresponds to a 

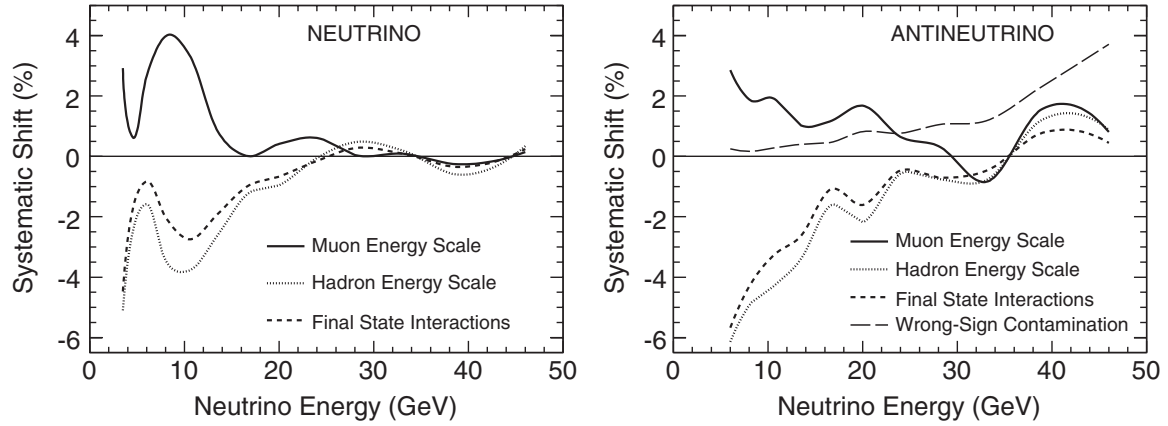

FIG. 8. Effect of the energy scale uncertainty on the neutrino (left panel) and antineutrino (right panel) extracted cross section. The curves give the shape distortion due to a one-sided $1 \sigma$ error. The solid lines show the effect of increasing the muon energy scale by $2 \%$ for stopping muons and $4 \%$ for exiting muons. The dotted lines show the effect of increasing the hadronic energy scale by $5.6 \%$, and the dashed lines show the effect of shifting the final state interaction model. The plot on the right also shows the effect of wrong-sign contamination uncertainty (large dashes) on the antineutrino cross section.

change of less than $1 \%$, which we take to be the $\mathrm{NC}$ contamination uncertainty.

To account for uncertainties in the acceptance correction, which arise from modeling of detector geometry, alignment, and magnetic field, we collected a dedicated data set with the detector magnetic field polarity reversed (set to focus positive charges) from its nominal running mode which focuses negative charges. In this data set, muon tracks pass through a different region of the detector and previously focused tracks bend away from the coil region. The flux and cross section are extracted and are compared with their values measured in the nominal mode. The flux sample relies more heavily on the muon track measurement than does the cross section sample and consequently larger effects are seen in the measured flux (on average $5 \%$ for neutrino and $2.5 \%$ for antineutrino flux). Smaller differences are expected for the antineutrino sample because more of the tracks exit the detector in the downstream region and do not pass near the difficult-tomodel coil region in either mode. A systematic uncertainty on the nominal polarity flux is taken to be half the difference between it and the flux extracted in the reversed polarity sample. This is added in quadrature with the other uncertainties. The cross section sample relies less heavily on the track momentum. The differences in measured cross section are at the level of $1 \%$, which are neglected.

Since the cross section model is used to apply a small energy dependent correction to the flux sample [see Eq. (5)], we take into account uncertainties in the model parameters described in Sec. II C. We account for uncertainties in the quasielastic and resonance contributions to the cross section by varying the axial mass parameters $M_{A}^{Q E}$ and $M_{A}^{R E S}$ in our model by $\pm 15 \%$. The resulting effect on the cross section is less than $2 \%$. In this measurement, we include an additional uncertainty in the DIS component of the model to account for contributions to the $\nu$ dependence of the cross section that could affect the flux extraction. To quantify the resulting uncertainty, we vary each parameter in the model [34] and study the change of the reduced $\chi^{2}$ of the fit to the charged lepton data from which they were originally determined. We take the shift that corresponds to a one unit shift in fit $\chi^{2}$ as the uncertainty for each parameter. The values and the associated uncertainties of $A_{h t}, B_{h t}, C_{v 1 u}$, and $C_{v 2 u}$ are determined to be $0.538 \pm 0.134,0.305 \pm 0.076,0.291 \pm 0.087$, and $0.189 \pm 0.076$, respectively. The other parameters $\left(C_{v 1 d}, C_{v 2 d}, C_{s u}\right.$, and $\left.C_{s d}\right)$ have a negligible effect on the analysis. The effects of these uncertainties on the flux
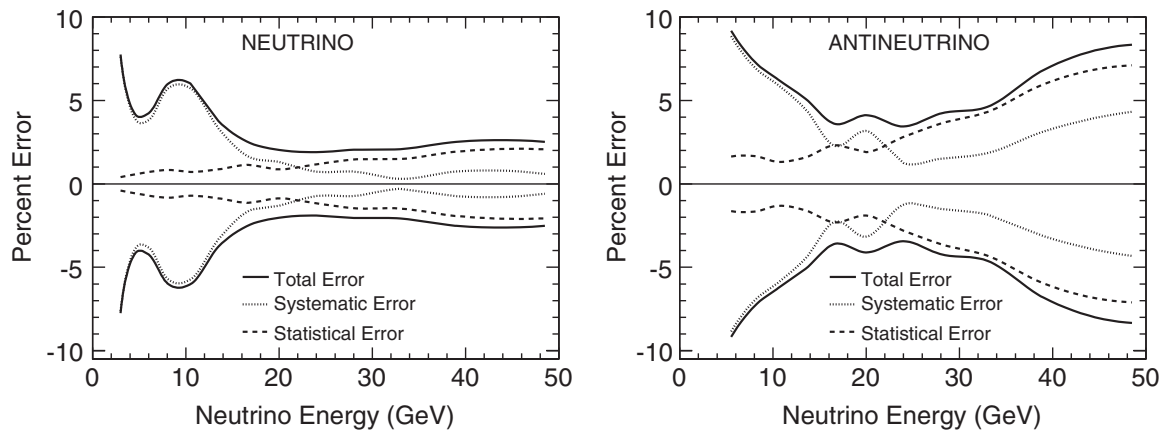

FIG. 9. Summary of the statistical, total systematic, and total uncertainty for the neutrino (left panel) and antineutrino (right panel) extracted cross sections. 
measurement and the acceptance correction are propagated to an uncertainty on the extracted cross section. The contributions from each parameter shift are added linearly to form the total DIS model uncertainty which is $2 \%$ below $8 \mathrm{GeV}$ for both neutrino and antineutrino cross sections, and is negligible above this energy.

The contamination from wrong-sign events is significant only for the antineutrino sample. To evaluate the uncertainty from this source, we recompute the cross section assuming no wrong-sign contamination and 2 times as much wrong-sign contamination. The resulting uncertainty is negligible below $15 \mathrm{GeV}$ but is about $4 \%$ at the highest energies.

The systematic, statistical, and total uncertainties for the neutrino and antineutrino cross sections are summarized in Fig. 9.

In the cross section ratio significant additional cancellation of uncertainties occurs. At the lower energies the uncertainties are about half those shown in Fig. 8. At higher energies they are $1 \%$ to $2 \%$ each, except for the wrong-sign contamination which is significant only for the antineutrino sample.

\section{RESULTS}

Figure 10 shows the extracted energy dependence of the total cross section divided by energy $(\sigma / E)$ for $\nu_{\mu} N$ CC and for $\bar{\nu}_{\mu} N$ CC interactions on an isoscalar target. The cross section values are assigned to the average energy in the bin. Both cross sections approach a linear energy dependence for energies above $20 \mathrm{GeV}$. For neutrinos $\sigma / E$ drops with increasing energy in the lower energy region. At $3 \mathrm{GeV}$ the quasielastic cross section is still expected to be appreciable $(\approx 15 \%)$. Its contribution to $\sigma / E$ falls rapidly with increasing energy as inelastic processes (resonance production and DIS) turn on. For antineutrinos the measured $\sigma / E$ rises gradually in the region 5-20 GeV to its asymptotic high-energy value. In this case the falling fractional contribution of the quasielastic cross section is offset by the more gradual turn-on of the DIS process, which is expected due to its strong dependence on the antiquark component which rises slowly with increasing $Q^{2}$. Table III summarizes the neutrino and antineutrino cross section results.

Figure 11 shows MINOS neutrino and antineutrino results compared to the results from other experiments. The MINOS neutrino cross section agrees with previous measurements from CRS [9], SKAT [14], IHEP-JINR [12], and GGM-PS [10], but these experiments have significantly larger uncertainties. Our neutrino cross section is in good agreement and has comparable precision with the recent NOMAD measurement [15]. Our result is systematics limited in the region below $15 \mathrm{GeV}$ where the largest uncertainties come from knowledge of the absolute muon and hadronic energy scales, whereas in NOMAD the flux determination dominates the uncertainty. The MINOS antineutrino cross section result is in good agreement with the sparse data available at lower energies and has much smaller uncertainty in the $10-30 \mathrm{GeV}$ region.

Figure 12 and Table IV show the ratio of the $\bar{\nu}_{\mu} N \mathrm{CC}$ to $\nu_{\mu} N$ CC inclusive cross section as a function of energy. Because of the cancellation of many of the systematic uncertainties the MINOS result is statistics limited above $10 \mathrm{GeV}$. The cross section ratio appears to gradually approach its asymptotic scaling value of $0.504 \pm 0.003$, defined by the world average from $30-200 \mathrm{GeV}$ calculated from previous experiments [4]. The MINOS average ratio measured from $30-50 \mathrm{GeV}$ of $0.489 \pm 0.012$ is in good

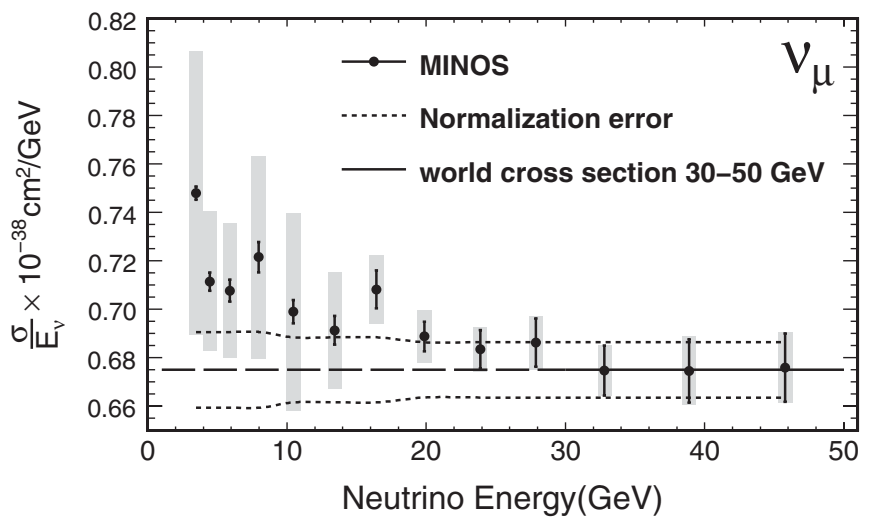

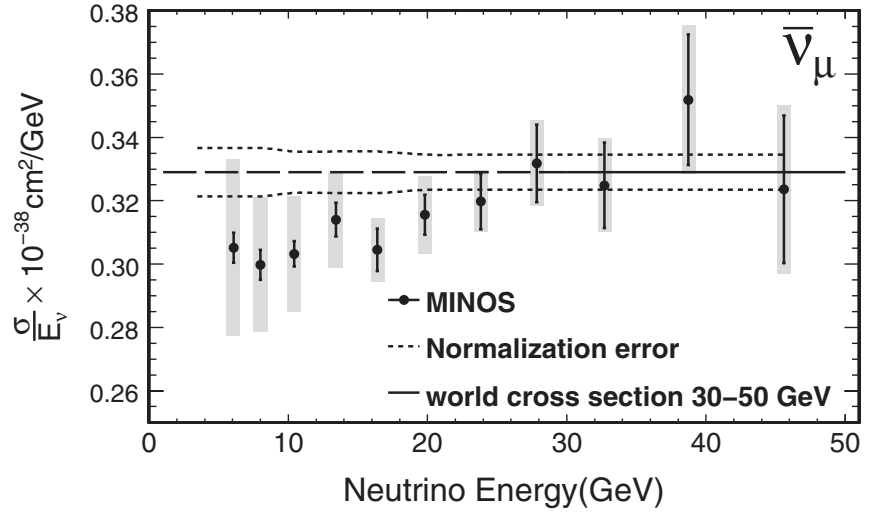

FIG. 10. Neutrino (left panel) and antineutrino (right panel) charged-current inclusive cross section per nucleon divided by energy for an isoscalar iron target. The black error bars show the statistical uncertainty and the shaded boxes show the statistical and systematic uncertainties added in quadrature. The dotted bands show the uncertainty on the normalization (about $1.5 \%$ ). The solid black lines show the world average neutrino cross section value of $0.675 \times 10^{-38} \mathrm{~cm}^{2} / \mathrm{GeV}$ from 30 to $50 \mathrm{GeV}$ [1-3,11,19] and the dashed black lines show this value extrapolated to lower energies. The neutrino cross section above $30 \mathrm{GeV}$ is normalized using this world average value and the same normalization constant is then applied to the antineutrinos. The solid black line on the antineutrino cross section plot shows a world average antineutrino cross section value of $0.329 \times 10^{-38} \mathrm{~cm}^{2} / \mathrm{GeV}$ from 30 to $50 \mathrm{GeV}$ [2,3,11]. This value is shown for comparison and is not used for antineutrino sample normalization. 
TABLE III. Summary of neutrino (left) and antineutrino (right) cross section results. The second column for each species shows the average energy in each bin. The uncertainties shown in columns 4-7 for each species are the statistical, systematic, normalization, and total contributions, respectively. The total uncertainty is obtained by summing the statistical, systematic, and normalization uncertainties in quadrature.

\begin{tabular}{|c|c|c|c|c|c|c|c|c|c|c|c|c|}
\hline \multirow{2}{*}{$\begin{array}{l}E \text { bin } \\
(\mathrm{GeV}) \\
3-4\end{array}$} & $\left\langle E_{\nu}\right\rangle$ & $\sigma / E$ & Stat. error & \multicolumn{2}{|c|}{$\begin{array}{l}\text { Neutrino } \\
\text { Syst. error Norm. error } \\
\left(10^{-38} \mathrm{~cm}^{2} / \mathrm{GeV}\right)\end{array}$} & Total error & \multirow[t]{2}{*}{$\begin{array}{c}\left\langle E_{\bar{\nu}}\right\rangle \\
(\mathrm{GeV})\end{array}$} & \multirow[t]{2}{*}{$\sigma / E$} & Stat. error & $\begin{array}{l}\text { Antineutrino } \\
\text { Syst. error } \\
\left(10^{-38} \mathrm{~cm}\right.\end{array}$ & $\begin{array}{l}\text { Norm. error } \\
\text { (GeV) }\end{array}$ & Total error \\
\hline & 3.48 & 0.748 & 0.003 & 0.058 & 0.017 & 0.061 & & & & & & \\
\hline $4-5$ & 4.45 & 0.711 & 0.004 & 0.029 & 0.017 & 0.033 & & & & & & \\
\hline $5-7$ & 5.89 & 0.708 & 0.005 & 0.027 & 0.016 & 0.032 & 6.07 & 0.305 & 0.005 & 0.027 & 0.007 & 0.029 \\
\hline 7-9 & 7.97 & 0.722 & 0.006 & 0.041 & 0.017 & 0.045 & 7.99 & 0.300 & 0.005 & 0.021 & 0.007 & 0.022 \\
\hline 9-12 & 10.45 & 0.699 & 0.005 & 0.041 & 0.014 & 0.043 & 10.43 & 0.303 & 0.004 & 0.018 & 0.006 & 0.019 \\
\hline $12-15$ & 13.43 & 0.691 & 0.006 & 0.023 & 0.014 & 0.028 & 13.42 & 0.314 & 0.005 & 0.014 & 0.006 & 0.016 \\
\hline $15-18$ & 16.42 & 0.708 & 0.008 & 0.012 & 0.014 & 0.020 & 16.41 & 0.304 & 0.007 & 0.007 & 0.006 & 0.012 \\
\hline $18-22$ & 19.87 & 0.689 & 0.006 & 0.009 & 0.012 & 0.016 & 19.82 & 0.316 & 0.006 & 0.011 & 0.005 & 0.013 \\
\hline $22-26$ & 23.88 & 0.683 & 0.008 & 0.005 & 0.012 & 0.015 & 23.82 & 0.320 & 0.009 & 0.004 & 0.005 & 0.011 \\
\hline $26-30$ & 27.89 & 0.686 & 0.010 & 0.004 & 0.012 & 0.016 & 27.84 & 0.332 & 0.012 & 0.005 & 0.006 & 0.015 \\
\hline $30-36$ & 32.81 & 0.675 & 0.010 & 0.002 & 0.011 & 0.016 & 32.72 & 0.325 & 0.014 & 0.006 & 0.005 & 0.016 \\
\hline $36-42$ & 38.87 & 0.675 & 0.013 & 0.005 & 0.011 & 0.018 & 38.74 & 0.352 & 0.021 & 0.011 & 0.006 & 0.024 \\
\hline $42-50$ & 45.77 & 0.676 & 0.014 & 0.004 & 0.011 & 0.019 & 45.61 & 0.324 & 0.023 & 0.013 & 0.005 & 0.027 \\
\hline
\end{tabular}

agreement with the asymptotic value. At $10 \mathrm{GeV}$ the measured ratio is $14 \%$ below the asymptotic value with $6.6 \sigma$ significance and at $24 \mathrm{GeV}$ the measurement lies $7 \%$ below with $2.4 \sigma$ significance. Our precise data show a slower approach to scaling behavior than has been previously claimed by low-energy measurements, ${ }^{2}$ which found their data to be consistent with scaling in the few $\mathrm{GeV}$ range [8,14,22].

Figure 12 also shows the cross section ratio compared with the few other existing measurements. The MINOS data uniquely span the $10-30 \mathrm{GeV}$ region. It overlaps the precise high-energy measurements $[17,21]$ as well as the Gargamelle low-energy measurement [22] which has precision of only about $20 \%$.

The total neutrino and antineutrino cross section in the quark parton model, which describes neutrino scattering at high energy, can be written as

$$
\sigma(\nu N)=\frac{G_{F}^{2} M E}{\pi}\left(Q+\frac{1}{3} \bar{Q}\right),
$$

and

$$
\sigma(\bar{\nu} N)=\frac{G_{F}^{2} M E}{\pi}\left(\bar{Q}+\frac{1}{3} Q\right)
$$

with the ratio of the two given by

$$
r=\frac{\sigma(\bar{\nu} N)}{\sigma(\nu N)}=\frac{1+3 \bar{Q} / Q}{3+\bar{Q} / Q},
$$

where $Q=\int x[u(x)+d(x)] d x \quad$ and $\quad \bar{Q}=\int x[\bar{u}(x)+$ $\bar{d}(x)] d x$. Here $u(x)[\bar{u}(x)]$ and $d(x)[\bar{d}(x)]$ are the parton

\footnotetext{
${ }^{2}$ These are based on fits to the measured neutrino cross section with energy.
}

distribution functions (PDFs) for the up and down quarks (antiquarks) in the nucleon, respectively. In the limit of large $Q^{2}, Q^{2} \gg M^{2}$, the PDFs depend only on $x$ and are independent of $Q^{2}$. In this limit the QPM predicts scaling behavior, i.e., a linear dependence of the cross sections with energy. In the low-energy (low $Q^{2}$ ) limit, scaling violations occur and the QPM breaks down. Scattering off the entire nucleon (quasielastic scattering) and resonance production, where the nucleon is excited and decays

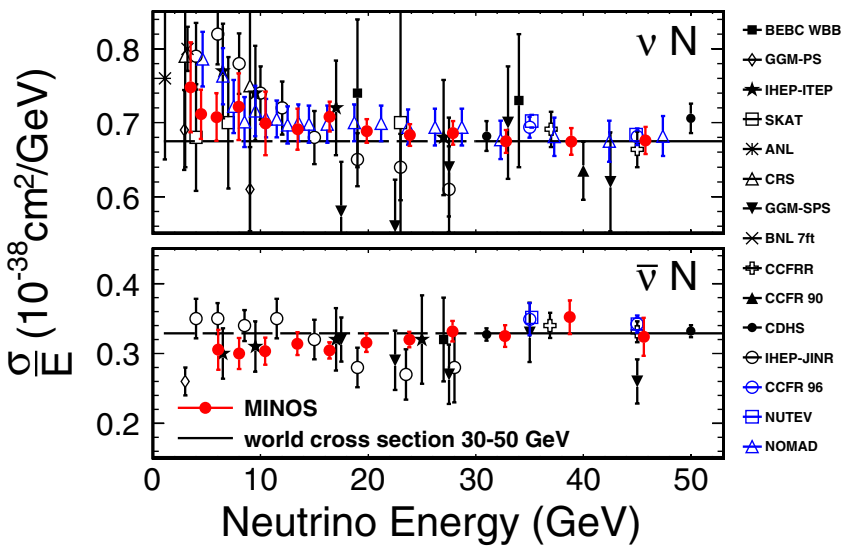

FIG. 11 (color online). MINOS neutrino and antineutrino charged-current inclusive cross section compared with other experimental results [1-3,8-13,15,17,19-21,46,47]. The error bars show the statistical, systematic, and normalization uncertainties added in quadrature. The solid black line shows the average world cross section in the 30 to $50 \mathrm{GeV}$ region for the neutrino $\left(0.675 \times 10^{-38} \mathrm{~cm}^{2} / \mathrm{GeV}\right)$ and the antineutrino $\left(0.329 \times 10^{-38} \mathrm{~cm}^{2} / \mathrm{GeV}\right)$. The curve is extrapolated to the $<30 \mathrm{GeV}$ region (dashes) to guide the eye. 

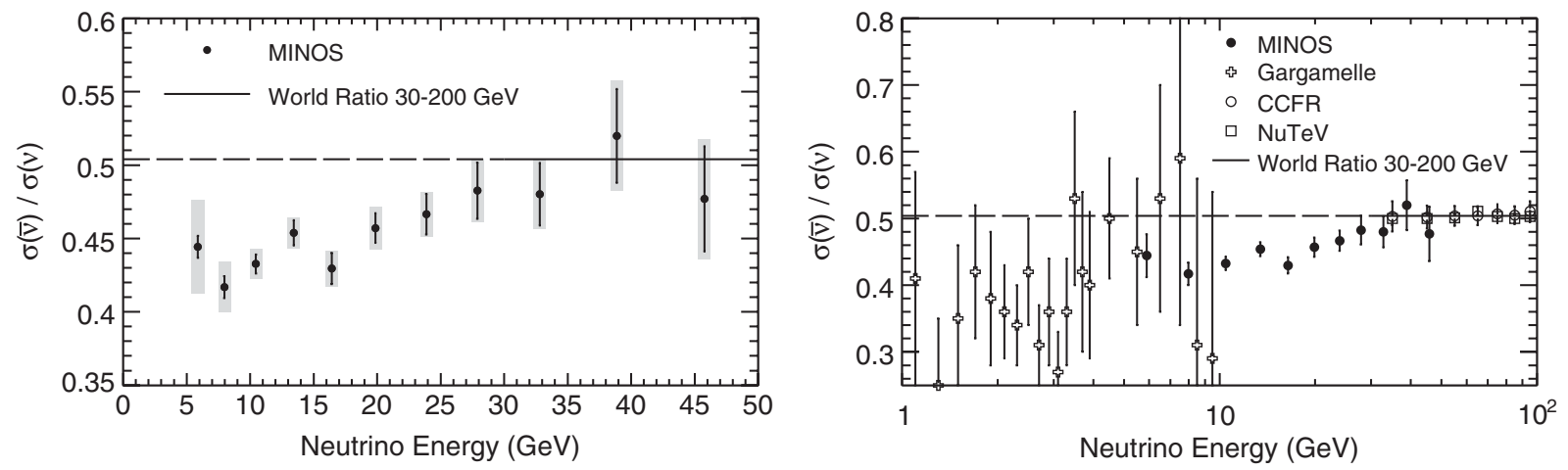

FIG. 12. (Left panel) Ratio of antineutrino-nucleon to neutrino-nucleon cross section as a function of energy. Black error bars show the statistical uncertainty and shaded boxes show the total uncertainty with statistical and systematic uncertainties added in quadrature. The solid black line at $0.504 \pm 0.003$ is drawn at the average value obtained from previous measurements over the energy range $30-$ $200 \mathrm{GeV}$ [4]. (Right panel) Comparison of measured $r$ with other measurements for $E<100 \mathrm{GeV}$. The MINOS result spans the intermediate-energy range and overlaps with the low-energy data [22] as well as with precise high-energy measurements [17,21].

to low multiplicity final states, must also be considered to account for the energy dependence of the cross section.

The ratio $r$ is constant with energy in the QPM and depends only on the integrated quark and antiquark distributions in the high- $Q^{2}$ limit. Equation (9) indicates antiquarks are relatively more important in the antineutrino scattering case. $r$ approaches the limiting value of $1 / 3$ if antiquarks are not present in the nucleon. High-energy measurements of $r$ can be used to measure the fraction of momentum carried by antiquarks in the nucleon.

In order to interpret our measurement of $r$ in the context of the QPM the quasielastic contribution is removed from the measured value by defining $r_{\text {inel }}=\sigma_{\text {inel }}^{\bar{\nu}} / \sigma_{\text {inel }}^{\nu}$ to be the cross section ratio for the purely inelastic contribution to

TABLE IV. The measured cross section ratio $r$ at the bin average energy along with statistical and total uncertainties is given. To compute $r_{\text {inel }}$, the NEUGEN3 [23] cross section model is used to remove the fractional quasielastic contribution. The quark parton model is used to estimate the fraction of the total quark momentum that is carried by antiquarks, $\frac{\bar{Q}}{Q+\bar{Q}}$. Uncertainties computed for $\Delta r_{\text {inel }}$ and the antiquark fraction do not include any model uncertainty contributions.

\begin{tabular}{lccccccc}
\hline \hline $\begin{array}{l}\text { Energy } \\
(\mathrm{GeV})\end{array}$ & $r$ & Stat. err. Total err. & $r_{\text {inel }}$ & $\Delta r_{\text {inel }}$ & $\frac{\bar{Q}}{Q+\bar{Q}}$ & $\Delta\left(\frac{\bar{Q}}{Q+\bar{Q}}\right)$ \\
\hline 5.9 & 0.444 & 0.007 & 0.032 & 0.407 & 0.029 & 0.079 & 0.030 \\
8.0 & 0.417 & 0.008 & 0.016 & 0.389 & 0.016 & 0.060 & 0.016 \\
10.5 & 0.433 & 0.006 & 0.010 & 0.410 & 0.010 & 0.081 & 0.010 \\
13.4 & 0.454 & 0.009 & 0.010 & 0.435 & 0.010 & 0.106 & 0.010 \\
16.4 & 0.430 & 0.011 & 0.012 & 0.415 & 0.012 & 0.086 & 0.012 \\
19.9 & 0.457 & 0.010 & 0.015 & 0.444 & 0.014 & 0.115 & 0.014 \\
23.9 & 0.467 & 0.014 & 0.015 & 0.455 & 0.015 & 0.126 & 0.014 \\
27.9 & 0.482 & 0.019 & 0.022 & 0.472 & 0.021 & 0.142 & 0.019 \\
32.8 & 0.480 & 0.021 & 0.023 & 0.472 & 0.023 & 0.141 & 0.021 \\
38.9 & 0.520 & 0.032 & 0.037 & 0.512 & 0.037 & 0.177 & 0.032 \\
45.8 & 0.477 & 0.036 & 0.041 & 0.471 & 0.040 & 0.140 & 0.037 \\
\hline \hline
\end{tabular}

the cross section. To compute $r_{\text {inel }}$, the NEUGEN3 cross section model [23] is used to remove the fractional quasielastic contribution. ${ }^{3}$ Table IV gives the measured ratio $r$ and the inelastic fraction $r_{\text {inel }}$ along with their experimental uncertainties. The similarly slow increase of $r_{\text {inel }}$ with energy shows that the decrease in the quasielastic contributions alone has only a small effect on the observed shape.

Equation (10) can be rearranged to give the fraction of total quark momentum in the nucleon that is carried by antiquarks, $\frac{\bar{Q}}{Q+\bar{Q}}=\frac{1}{2} \frac{(3 r-1)}{(r+1)}$. This fraction as a function of energy is also given in Table IV. As neutrino energy decreases, one moves increasingly away from the domain of validity of this expression, which is derived in the DIS region. Target mass corrections as well as higher-twist terms become more important, especially at high $x$. However, the high- $x$ region contributes little to the $Q$ and $\bar{Q}$ integrals. In the approximation in which the contributions from these effects are small, our results are consistent with a nonzero antiquark content in the nucleon at our lowest energy, $5.9 \mathrm{GeV}\left(\left\langle Q^{2}\right\rangle=1.4 \mathrm{GeV}^{2}\right)$ and a gradual increase of the antiquark fraction with energy. In order to accurately extract the antiquark fraction from our data, a full higher order QCD model that incorporates these effects is required.

\section{CONCLUSION}

We have measured the charged-current neutrino-nucleus inclusive cross section in the energy range $3-50 \mathrm{GeV}$ with a precision of $2 \%-8 \%$ and the antineutrino-nucleus cross

\footnotetext{
${ }^{3}$ The NEUGEN3 model, as described earlier, uses a value of $M_{A}^{Q E}=0.99$. We provide the raw measured $r$ so that one can use other models to compute the inelastic fraction. This will be especially useful as knowledge of $M_{A}^{Q E}$ improves. For reference, increasing $M_{A}^{Q E}$ by 0.15 decreases the inelastic fraction by less than $1 \%$ at $5.9 \mathrm{GeV}$, which is small compared with the experimental uncertainty.
} 
section from 5-30 GeV with a precision in the range 3\%$9 \%$. The flux was determined by using a subsample of lowhadronic-energy events to measure the flux shape and the world average cross section above $30 \mathrm{GeV}$ for normalization. This method was previously used at higher energies $[17,21]$ and here we have extended it down to $3 \mathrm{GeV}$. While the measurements are systematics dominated, the overall systematic uncertainty benefits from partial cancellation in detector related systematic uncertainties that arise from measuring the flux and the $\mathrm{CC}$ event rate in the same detector. Both measurements impact the precision of total cross section measurements in the less than $30 \mathrm{GeV}$ range.

Our measurement of the antineutrino to neutrino cross section ratio is the most precise in the less than $30 \mathrm{GeV}$ range, where only one previous measurement has been performed [22]. The measured rise of the cross section ratio with energy is consistent with an expected slow rise in the antineutrino inelastic cross section with the increase in number of sea-quark degrees of freedom for increasing $Q^{2}$.

The measurement presented here can be used to tune neutrino and antineutrino cross section models which benefit ongoing and future neutrino oscillation measurements.

\section{ACKNOWLEDGMENTS}

This work was supported by the U.S. DOE; the UK STFC; the U.S. NSF; the State and University of Minnesota; the University of Athens, Greece; and Brazil's FAPESP and CNPq. We are grateful to the Minnesota Department of Natural Resources, the crew of the Soudan Underground Laboratory, and the staff of Fermilab for their contribution to this effort.

\section{APPENDIX: UNCORRECTED DATA SAMPLE}

Our measurement has cross section model dependence which arises from the correction for the minimum muon energy requirement $E_{\mu}>1.5 \mathrm{GeV}$ in the cross section sample $\left[N_{\mathrm{CC}}^{\nu(\bar{\nu})}(E)\right]$ and for the small energy dependence in the low- $\nu$ flux sample [see Eq. (2)]. Here we provide the raw data cross section to flux sample ratio $R^{\nu(\bar{\nu})}$ corrected only for detector effects and backgrounds. This will allow the reader to use an alternative cross section model and our data to compute neutrino and antineutrino cross sections.

Table $\mathrm{V}$ gives the values of $R^{\nu}$ ( $R^{\bar{\nu}}$ for antineutrinos) where both numerator and denominator have been corrected for detector effects and backgrounds using our GEANT3-based detector simulation. The ratio has not been corrected for the effect of the kinematic $E_{\mu}>1.5 \mathrm{GeV}$ cut, which affects only the numerator. The unnormalized neutrino cross section $\sigma_{\text {unnorm }}^{\nu}(E)$ can be computed from $R^{\nu}(E)$ by applying two corrections,

$$
\sigma_{\text {unnorm }}^{\nu}(E)=K^{\nu}(E) \times S^{\nu}\left(\nu_{0}, E\right) \times R^{\nu}(E),
$$

where $K^{\nu}(E)=N(E) / N\left(E, E_{\mu}>1.5 \mathrm{GeV}\right)$ is the ratio of total cross section events for all muon energies to that with muon energies larger than $1.5 \mathrm{GeV}$ in each energy bin, and $S^{\nu}\left(\nu_{0}, E\right)$ is defined in Eq. (5), (where $\nu_{0}=1,2$ or $5 \mathrm{GeV}$ ).

The cross section is normalized using the values of $R^{\nu}$ for different $\nu_{0}$ cut samples provided in Table VI. To improve statistical precision of the flux sample three different values of the $\nu_{0}$ cut were used in the analysis: $\nu_{0}<$ $1 \mathrm{GeV}$ applies for the neutrino energies $E<9 \mathrm{GeV}, \nu_{0}<$ $2 \mathrm{GeV}$ for $9<E<18 \mathrm{GeV}$, and $\nu_{0}<5 \mathrm{GeV}$ for $E>$ $18 \mathrm{GeV}$. We define the normalization constant $\operatorname{Norm}\left(\nu_{0}\right)$ for each $\nu_{0}$ sample as

$$
\sigma_{\text {norm }}^{\nu}(E)=\operatorname{Norm}\left(\nu_{0}\right) \times \sigma_{\text {unnorm }}^{\nu}(E) .
$$

$\operatorname{Norm}\left(\nu_{0}\right)$ is obtained by first computing unnormalized cross sections in the range $30<E_{\nu}<50 \mathrm{GeV}$ using $R^{\nu}$ from Table VI and Eq. (A1). The weighted average of $\sigma_{\text {unnorm }}^{\nu}(E) /\left\langle E_{\nu}\right\rangle$ in this energy range is computed for each $\nu_{0}$ sample using the statistical errors on $R^{\nu}$ also given

TABLE V. Ratio of cross section to flux sample where both numerator and denominator have been corrected for detector effects and backgrounds. The text describes how to use these data and a cross section model to compute neutrino and antineutrino cross sections.

\begin{tabular}{lcc|cc}
\hline \hline & \multicolumn{2}{c|}{ Neutrino } & \multicolumn{2}{c}{ Antineutrino } \\
$E$ bin $(\mathrm{GeV})$ & $\left\langle E_{\nu}\right\rangle(\mathrm{GeV})$ & $R^{\nu}$ & $\left\langle E_{\bar{\nu}}\right\rangle(\mathrm{GeV})$ & $R^{\bar{\nu}}$ \\
\hline $3-4$ & 3.48 & 1.72 & & \\
$4-5$ & 4.45 & 2.35 & & \\
$5-7$ & 5.89 & 3.31 & 6.07 & 2.22 \\
$7-9$ & 7.97 & 4.93 & 7.99 & 2.78 \\
$9-12$ & 10.5 & 3.71 & 10.4 & 2.10 \\
$12-15$ & 13.4 & 4.87 & 13.4 & 2.69 \\
$15-18$ & 16.4 & 6.22 & 16.4 & 3.11 \\
$18-22$ & 19.9 & 3.32 & 19.8 & 1.90 \\
$22-26$ & 23.9 & 3.98 & 23.8 & 2.22 \\
$26-30$ & 27.9 & 4.68 & 27.8 & 2.60 \\
$30-36$ & 32.8 & 5.45 & 32.7 & 2.94 \\
$36-42$ & 38.9 & 6.49 & 38.7 & 3.75 \\
$42-50$ & 45.8 & 7.70 & 45.6 & 4.04 \\
\hline \hline
\end{tabular}

TABLE VI. Raw ratio of cross section to flux sample (as in Table V) in the normalization region $(30<E<50 \mathrm{GeV})$. The separate $R^{\nu}$ columns give the ratio for the three different $\nu_{0}$ cut values. Each sample is separately normalized using the corresponding $E>30 \mathrm{GeV}$ data points.

\begin{tabular}{lcccc}
\hline \hline$E_{\nu}(\mathrm{GeV})\left\langle E_{\nu}\right\rangle(\mathrm{GeV})$ & \multicolumn{3}{c}{$R^{\nu}$} \\
& & $\nu_{0}<1 \mathrm{GeV}$ & $\nu_{0}<2 \mathrm{GeV}$ & $\nu_{0}<5 \mathrm{GeV}$ \\
\hline $30-36$ & 32.8 & $22.1 \pm 0.6$ & $12.3 \pm 0.3$ & $5.5 \pm 0.1$ \\
$36-42$ & 38.9 & $26.0 \pm 0.9$ & $14.5 \pm 0.4$ & $6.5 \pm 0.1$ \\
$42-50$ & 45.8 & $30.5 \pm 1.2$ & $17.2 \pm 0.5$ & $7.7 \pm 0.2$ \\
\hline \hline
\end{tabular}


in Table VI. $\operatorname{Norm}\left(\nu_{0}\right)$ is then obtained by scaling this to the world average value $0.675 \times 10^{-38} \mathrm{~cm}^{2} / \mathrm{GeV}$.

The same overall normalization constants are used to obtain the antineutrino cross section

$$
\begin{aligned}
\sigma_{\text {norm }}^{\bar{\nu}}(E)= & {\left[\operatorname{Norm}\left(\nu_{0}\right) \times G^{\text {corr }}\left(\nu_{0}\right)\right] \times K^{\bar{\nu}}(E) \times S^{\bar{\nu}}\left(\nu_{0}, E\right) } \\
& \times R^{\bar{\nu}}(E) .
\end{aligned}
$$

The additional normalization factor $G^{\text {corr }}\left(\nu_{0}\right)$ is used to account for a small difference in neutrino and antineutrino $F_{2}$ structure functions (see Sec. III C). $G^{\text {corr }}\left(\nu_{0}\right)$ can be computed from the ratio of asymptotic values of antineu- trino to neutrino low- $\nu$ cross sections,

$$
G^{\mathrm{corr}}\left(\nu_{0}\right)=\frac{\sigma^{\bar{\nu}}\left(\nu<\nu_{0}, E \rightarrow \infty\right)}{\sigma^{\nu}\left(\nu<\nu_{0}, E \rightarrow \infty\right)}
$$

for the three different $\nu_{0}$ values.

The uncertainty in the new measured cross section should be estimated using the fractional cross section uncertainty (syst $\oplus$ stat) given in Table III, which properly takes into account cancellations in several systematic uncertainties in the cross section and flux samples.
[1] P. Auchincloss et al., Z. Phys. C 48, 411 (1990).

[2] D. Macfarlane et al., Z. Phys. C 26, 1 (1984).

[3] P. Berge et al., Z. Phys. C 35, 443 (1987).

[4] C. Amsler et al., Phys. Lett. B 667, 1 (2008).

[5] R. Feynman, Phys. Rev. Lett. 23, 1415 (1969).

[6] D. Ayres et al. (Nova Collaboration), arXiv:hep-ex/ 0210005.

[7] Y. Itow et al. (T2K Collaboration), aXiv:hep-ex/0106019.

[8] N. Baker et al., Phys. Rev. D 25, 617 (1982).

[9] C. Baltay et al., Phys. Rev. Lett. 44, 916 (1980).

[10] S. Campolillo et al., Phys. Lett. 84B, 281 (1979).

[11] J. Morfin et al., Phys. Lett. 104B, 235 (1981).

[12] V. Anikeev et al., Z. Phys. C 70, 39 (1996).

[13] A. Vovenko et al., Sov. J. Nucl. Phys. 30, 527 (1979).

[14] D. Baranov et al., Phys. Lett. 81B, 255 (1979).

[15] Q. Wu et al., Phys. Lett. B 660, 19 (2008).

[16] P. Astier et al. (NOMAD Collaboration), Nucl. Instrum. Methods Phys. Res., Sect. A 515, 800 (2003).

[17] W. Seligman, Ph.D. thesis, Columbia University, 1997), Nevis 292.

[18] S. R. Mishra, in Proceedings of the Workshop on Hadron Structure Functions and Parton Distributions, edited by D. Geesaman et al. (World Scientific, Singapore, 1990), pp. 84-123.

[19] D. Colley et al., Z. Phys. C 2, 187 (1979).

[20] O. Erriquez et al., Phys. Lett. 80B, 309 (1979).

[21] M. Tzanov et al., Phys. Rev. D 74, 012008 (2006).

[22] T. Eichten et al. (Gargamelle Collaboration), Phys. Lett. 46B, 274 (1973).

[23] H. Gallagher, Nucl. Phys. B, Proc. Suppl. 112, 188 (2002).

[24] C. Andreopoulos et al., Nucl. Instrum. Methods Phys. Res., Sect. A 614, 87 (2010).

[25] B. T. Fleming et al. (CCFR Collaboration), Phys. Rev. Lett. 86, 5430 (2001).

[26] P. Adamson et al. (MINOS Collaboration), Phys. Rev. Lett. 101, 131802 (2008).
[27] P. Adamson et al. (MINOS Collaboration), Phys. Rev. D 77, 072002 (2008)

[28] D. Michael et al., Nucl. Instrum. Methods Phys. Res., Sect. A 596, 190 (2008).

[29] R. Brun et al., CERN Program Library, Long Writeup Report No. W5013, 1994.

[30] A. Ferrari, P. R. Sala, A. Fasso, and J. Ranft, CERN Technical Report No. CERN-2005-010, 2005.

[31] C. Llewellyn-Smith, Phys. Rep. 3, 261 (1972).

[32] R. Feynman, M. Kislinger, and F. Ravndal, Phys. Rev. D 3, 2706 (1971).

[33] D. Rein and L. Sehgal, Ann. Phys. (N.Y.) 133, 79 (1981).

[34] A. Bodek, I. Park, and U. K. Yang, Nucl. Phys. B, Proc. Suppl. 139, 113 (2005).

[35] Z. Koba, H. B. Nielsen, and P. Olesen, Nucl. Phys. B40, 317 (1972).

[36] T. Sjostrand, Comput. Phys. Commun. 82, 74 (1994).

[37] S. Dytman, H. Gallagher, and M. Kordosky, arXiv:hep-ex/ 0806.2119 .

[38] R. Merenyi et al., Phys. Rev. D 45, 743 (1992).

[39] D. S. Baranov et al., Yad. Fiz. 40, 1454 (1984).

[40] D. E. Groom, N. V. Mokhov, and S. I. Striganov, At. Data Nucl. Data Tables 78, 183 (2001).

[41] R. Ospanov, Ph.D. thesis, University of Texas, Austin [FERMILAB-THESIS-2008-04, 2008].

[42] P. Adamson et al. (MINOS Collaboration), Nucl. Instrum. Methods Phys. Res., Sect. A 556, 119 (2006).

[43] M. A. Kordosky, Ph.D. thesis, University of Texas, Austin [FERMILAB-THESIS-2004-34, 2004].

[44] D. Bhattacharya, Ph.D. thesis, University of Pittsburgh [FERMILAB-THESIS-2009-11].

[45] D. Bardin and V. Dokuchaeva, Report No. JINR-E2-86260, 1986.

[46] J. Allaby et al., Z. Phys. C 38, 403 (1988).

[47] S. Barish et al., Phys. Rev. D 19, 2521 (1979). 